\title{
Functional determinants, index theorems, and exact quantum black hole entropy
}

\author{
Sameer Murthy ${ }^{a}$ and Valentin Reys ${ }^{b}$ \\ ${ }^{a}$ Department of Mathematics, King's College London, \\ The Strand, London WC2R 2LS, U.K. \\ ${ }^{b}$ Nikhef theory group, Science Park 105, \\ 1098 XG Amsterdam, The Netherlands \\ E-mail: sameer.murthy@kcl.ac.uk, vreys@nikhef.nl
}

ABSTRACT: The exact quantum entropy of BPS black holes can be evaluated using localization in supergravity. An important ingredient in this program, that has been lacking so far, is the one-loop effect arising from the quadratic fluctuations of the exact deformation (the $Q \mathcal{V}$ operator). We compute the fluctuation determinant for vector multiplets and hyper multiplets around $Q$-invariant off-shell configurations in four-dimensional $\mathcal{N}=2$ supergravity with $A d S_{2} \times S^{2}$ boundary conditions, using the Atiyah-Bott fixed-point index theorem and a subsequent zeta function regularization. Our results extend the large-charge on-shell entropy computations in the literature to a regime of finite charges. Based on our results, we present an exact formula for the quantum entropy of BPS black holes in $\mathcal{N}=2$ supergravity. We explain cancellations concerning $\frac{1}{8}$-BPS black holes in $\mathcal{N}=8$ supergravity that were observed in arXiv:1111.1161. We also make comments about the interpretation of a logarithmic term in the topological string partition function in the low energy supergravity theory.

KeYwords: Supersymmetric gauge theory, Black Holes in String Theory, Black Holes, Supergravity Models

ArXiv EPrint: 1504.01400v2 


\section{Contents}

1 Introduction and summary: quantum entropy of supersymmetric black holes

2 The set up for the evaluation of quantum entropy

3 Gauge-fixing and the introduction of ghosts

4 Calculation of the one-loop determinant

5 Relation to previous results for the black hole entropy

6 Exact formulas for $\mathcal{N}=2$ quantum black hole entropy and the relation to topological strings

A Conventions

B Killing spinors of $A d S_{2} \times S^{2}$

C Transversally elliptic operators and the symbol of $D_{10}$

\section{Introduction and summary: quantum entropy of supersymmetric black holes}

Consider a supersymmetric black hole in a four-dimensional theory of supergravity in asymptotically flat space, coupled to $\left(n_{\mathrm{v}}+1\right)$ gauge fields, and carrying electric and magnetic charges $\left(q_{I}, p^{I}\right), I=0,1, \cdots n_{\mathrm{v}}$, under these gauge fields. The near-horizon configuration of such a black hole is itself a fully supersymmetric solution of the theory, and can be decoupled and studied in its own right as a consistent quantum gravitational system. The classical near-horizon field configuration, and the classical entropy of the black hole, are determined in terms of the black hole charges, according to the well-known attractor mechanism [1].

The attractor equations, as presented in [1] for a two-derivative theory of supergravity, followed from the BPS equations in the near-horizon region, and the entropy of the black hole was given by the Bekenstein-Hawking formula $[2,3]$. These ideas were generalized to theories including higher-derivative interactions in $[4,5]$, by using an off-shell formulation of supergravity, and by using the more general Bekenstein-Hawking-Wald entropy formula [68]. These methods have allowed us to completely understand the BPS black hole entropy for any theory of supergravity based on a local effective action.

There is a useful reformulation of the attractor mechanism that relies only on the existence of a bosonic $\mathrm{SL}(2) \times \mathrm{SU}(2)$ symmetry in the near-horizon region [9]. This symmetry 
fixes the value of all the fields up to undetermined constants - the geometry is $A d S_{2} \times S^{2}$ with overall size $v$, the gauge fields have a constant electric field strength $e_{*}^{I}$ on the $A d S_{2}$ factor and a constant magnetic flux on the 2 -sphere with charge $p^{I}$, and the scalar fields take constant values $u^{a}$. The classical equations of motion then take the form of the extremization equations for the constant parameters:

$$
\frac{\partial \mathcal{L}^{\mathrm{eff}}}{\partial v}=0, \quad \frac{\partial \mathcal{L}^{\mathrm{eff}}}{\partial u^{a}}=0, \quad \frac{\partial \mathcal{L}^{\mathrm{eff}}}{\partial e_{*}^{I}}=q_{I},
$$

where $\mathcal{L}^{\text {eff }}\left(v, e_{*}^{I}, u^{a}, q_{I}, p^{I}\right)$ is the local effective Lagrangian (possibly containing higherderivative interactions) integrated over the $S^{2}$ factor and evaluated on the near-horizon configuration. The Bekenstein-Hawking-Wald entropy of the black hole is then equal to the Legendre transform of the effective Lagrangian $\mathcal{L}^{\text {eff }}$ at the attractor values of the various fields determined by $(1.1):^{1}$

$$
S_{\mathrm{BH}}^{\text {class }}=-\pi q_{I} e_{*}^{I}-\left.\pi \mathcal{L}^{\text {eff }}\right|_{\text {attr. }} .
$$

The equations (1.1), (1.2) are a concise and elegant way to recast the classical entropy of BPS black holes as a variational principle in the near-horizon region.

To include the effect of quantum fluctuations of the supergravity fields on the BPS black hole entropy, [10] promotes the above variational principle to a functional integral, called the quantum entropy, over all the fields of the theory that asymptote to the attractor configuration specified by (1.1). More precisely, it is the expectation value of the Wilson line

$$
\exp \left(S_{\mathrm{BH}}^{\mathrm{qu}}(q, p)\right) \equiv W(q, p)=\left\langle\exp \left[-i q_{I} \oint_{\tau} A^{I}\right]\right\rangle_{\mathrm{AdS}_{2}}^{\text {finite }} .
$$

The angular brackets indicate an integration (with an appropriate measure) over all the field fluctuations weighted by the exponential of the Wilsonian effective action at some fundamental scale defining the theory such as the string scale, and the superscript denotes a regularization of the divergences that arise from the infinite volume of $A d S_{2}$.

Our goal here is the exact evaluation of this functional integral, for which we use the technique of supersymmetric localization applied to supergravity [11-16], a development that was seeded by the powerful application of such methods to supersymmetric field theory [17] (see [18, 19] and references therein for very recent reviews). As we shall discuss below, the localization technique reduces a complicated quantum functional integral to the evaluation of a related integral in the semi-classical limit, i.e. keeping only its classical and one-loop contributions. In the context of $\mathcal{N}=2$ supergravity coupled to matter multiplets, the reduction to a specific semi-classical integral was established in [12], wherein the classical part of the computation was performed. In this paper, we compute the oneloop determinants of the matter field (vector and hyper multiplets) fluctuations. As we

\footnotetext{
${ }^{1}$ The Bekenstein-Hawking-Wald entropy is sometimes referred to as the "classical" entropy because it relies on a local effective action. We stress that this action can include higher-derivative interactions, e.g. coming from integrating out the massive modes of the theory. Perhaps a better notation would be "Wilsonian entropy" — in contrast to the "exact entropy", defined in (1.3), that we study in this paper.
} 
shall see, this is an important ingredient in the localization recipe, in the absence of which the final result lacks consistency.

The starting point of the localization method to compute a functional integral like (1.3) is the existence of a fermionic symmetry $Q$ that is realized off-shell in the theory, and that squares to a compact $\mathrm{U}(1)$ symmetry. One deforms the Lagrangian by a positive-definite $Q$-exact term $\lambda Q \mathcal{V}$, with $\lambda \in \mathbb{R}^{\geq 0}$ and $\mathcal{V}$ an appropriately chosen fermionic functional. The exactness guarantees that the functional integral is independent of the deformation parameter $\lambda$. On taking the $\lambda \rightarrow \infty$ limit, the problem reduces to a semi-classical evaluation of the original integral over the critical points of $Q \mathcal{V} .^{2}$

The set of critical points, called the localization locus $\mathcal{M}_{Q}$, is a drastically reduced - often finite-dimensional - space compared to the infinite-dimensional field space that we begin with. The choice $\mathcal{V}=\int d^{4} x \sum_{i}\left(Q \psi_{i}, \psi_{i}\right)(x)$, where the summation runs over all fermions $\psi_{i}$ of the theory and (., .) is an appropriate positive-definite inner product in Euclidean signature, is particularly convenient. For this choice, the bosonic localization locus is the set of all solutions of $Q \psi_{i}=0$, i.e. the zero modes of $Q$. The operator $Q \mathcal{V}$ vanishes on this locus, and the final answer consists of an integral over the zero modes of $Q$ of the exponential of the full original action times the quadratic fluctuation determinant of the $Q \mathcal{V}$ operator around the localization locus.

In the black hole context, we choose a supercharge $Q$ such that $Q^{2}=L_{0}-J_{0}$, where $L_{0}$ is the $\mathrm{U}(1)$ rotation of the $A d S_{2}$ and $J_{0}$ is a rotation of the $S^{2}$ in the fixed asymptotic $A d S_{2} \times$ $S^{2}$ region. For a theory of $\mathcal{N}=2$ supergravity coupled to $n_{\mathrm{v}}$ physical vector multiplets, the conformal supergravity formalism $[23,24]$ provides an off-shell closure of the supersymmetry algebra. In this context, the localizing manifold is labelled by $\left(n_{\mathrm{v}}+1\right)$ real parameters $\left\{\phi^{I}\right\}$, $I=0, \cdots, n_{\mathrm{v}}$, and the result of localizing the functional integral (1.3) takes the form [12]: ${ }^{3}$

$$
W^{\mathrm{pert}}(q, p)=\int_{\mathcal{M}_{Q}} \prod_{I=0}^{n_{\mathrm{v}}} d \phi^{I} \exp \left(-\pi q_{I} \phi^{I}+4 \pi \operatorname{Im} F\left(\left(\phi^{I}+i p^{I}\right) / 2\right)\right) Z_{\operatorname{det}}\left(\phi^{I}\right),
$$

where $F\left(X^{I}\right)$ is the holomorphic prepotential of the $\mathcal{N}=2$ supergravity theory (suppressing for now the dependence of $F$ on the Weyl multiplet, which is taken to be fixed to its attractor value). This formula shares a number of interesting features with the OSV proposal [27], and it is part of the attempt to derive and refine this conjecture from the gravitational theory. Details of the comparison with the the original OSV proposal are given in $[12,13]$. We shall make a comparison with the related proposal of [28] in section 6 .

In this paper we focus on the determinant factor $Z_{\text {det }}$ in (1.4) which is the main remaining problem in the derivation of the exact gravitational quantum entropy formula. This factor $Z_{\text {det }}$ includes the measure factor arising from the intrinsic curvature of the localization manifold, as well as the 1-loop determinant of quadratic fluctuations of the

\footnotetext{
${ }^{2} \mathrm{~A}$ rigorous treatment of the above argument uses the methods of equivariant cohomology, and the result is given by the Duistermaat-Heckman-Berline-Vergne-Atiyah-Bott localization formula [20-22]. We shall follow the treatment of [17] where these methods are nicely explained in field theory language.

${ }^{3}$ The superscript on the left-hand side indicates that we will consider an all-order perturbation theory result around the leading saddle point. There may be additional non-perturbative contributions, for example from orbifold configurations [16, 25, 26].
} 
deformation action $Q \mathcal{V}$ around the localization manifold: $Z_{\text {det }}=Z_{\text {det }}^{\text {ind }} Z_{1 \text {-loop }}$. The mea-


set ourselves here is to compute the one-loop fluctuation determinant for the $Q \mathcal{V}$ operator for vector multiplets and hyper multiplets. The computation of the graviton and gravitini determinants is under progress [30]. We compute the determinant of the fluctuations of the fields in the theory normal to the localization manifold, at an arbitrary point $\phi^{I}$, focusing here on the dependence of this determinant on the charges and on the fields $\phi^{I}$ and ignoring overall numerical constants. A non-trivial dependence on $\phi^{I}$ means that the non-zero modes (under $Q$ ) of bosons and fermions do not cancel in the functional integral. As we will see, the dependence of the determinant on the fields $\phi^{I}$ appears only through the scale of the fluctuating geometry.

In the vector multiplet, the gauge-fixing condition does not commute with the offshell supersymmetry, and to treat this problem, we develop a formalism to treat BRST symmetries for vector multiplets consistent with the off-shell closure of the supersymmetry algebra. We do so using the standard rules of quantization for theories with multiple gauge invariances [31, 32]. Our results are applicable to four-dimensional $\mathcal{N}=2$ supergravity coupled to vector multiplets in any background that preserves some supersymmetry. In the case of the (deformed) 4-sphere, it agrees with the treatment of [17, 33]. In the $A d S_{2} \times S^{2}$ background, our formalism leads to a different algebra.

Our main results concerning black hole entropy are as follows. Firstly, the functional determinants of vector and hyper multiplets is given in the concise formula (4.28). In theories of $\mathcal{N}=2$ supergravity, taking the holomorphic prepotential as input, and an assumption about the induced measure (Equation (6.7)), we derive a perturbatively exact formula for the quantum entropy of $\frac{1}{2}$-BPS black holes expressed in Equations (6.2), (6.8). Then we explain some non-trivial cancellations in theories of extended supergravity that agrees with corresponding microscopic results. Finally, we make an observation concerning a term logarithmic in one of the localization coordinates in the exact entropy formula. There is a natural interpretation of this coordinate as the topological string coupling, thus relating to an existing prediction of $[28,34,35]$.

The plan of the paper is as follows. In section 2 we set up the formalism for the calculation of the functional integral. In section 3 we deal with the gauge invariance, the BRST cohomology, and the issue of how to combine it with the off-shell supersymmetry. In section 4 we compute the one-loop determinants of the matter multiplets using the zetafunction regularization. In section 5 we discuss large-charge expansions of our results, and compare them to previously obtained results. In the final section 6 , we present an exact formula for BPS black hole entropy in $\mathcal{N}=2$ supergravity, and comment on the relations to topological strings.

Note added. While this paper was being prepared for publication, we received communication from R. Gupta, Y. Ito, and I. Jeon of [36] that contains overlapping results. 


\section{The set up for the evaluation of quantum entropy}

In this section we set the stage for the determinant calculations presented in the later sections. We first review the formalism of off-shell $\mathcal{N}=2$ conformal supergravity in which we work. We then review BPS black hole solutions in the theory and the corresponding attractor equations. Choosing one supercharge $Q$, we review the localizing equations corresponding to $Q$, and the set of solutions, i.e. the localizing manifold. We then present the algebra of $Q$ as it acts on the various fluctuating fields of the theory.

\section{The conformal supergravity formalism and the classical black hole}

The $\mathcal{N}=2$ conformal supergravity $[23,24]$ is a formalism which allows for off-shell closure of supersymmetry transformations. The theory describes the Weyl multiplet coupled to $\left(n_{\mathrm{v}}+1\right)$ vector multiplets labelled by $I=0, \cdots, n_{\mathrm{v}}$. The Weyl multiplet includes the vierbein $e_{\mu}^{a}$, the gravitino fields $\psi_{\mu}^{i}$, an antisymmetric tensor $T_{a b}^{i j}$, as well as other fields needed to close the multiplet off-shell. The index $i=1,2$ is a fundamental of the $\mathrm{SU}(2)$ R-symmetry of the theory. Each vector multiplet contains a gauge field $A_{\mu}^{I}$, a complex scalar $X^{I}$, a real $\mathrm{SU}(2)$ triplet $Y_{i j}^{I}$ of auxiliary scalars, and the gaugini $\Omega_{i}^{I}$. In this paper, we will only consider abelian vector multiplets.

The supergravity action that we consider is specified by a holomorphic function called the prepotential $F\left(X^{I}, \widehat{A}\right)$, describing the coupling of the vector multiplets to the background Weyl multiplet through chiral-superspace integrals. ${ }^{4}$ Here, $X^{I}$ is the lowest component of the vector multiplet and $\widehat{A} \equiv\left(T_{\mu \nu}^{-}\right)^{2}$ is the lowest component of the chiral multiplet built as the square of the Weyl multiplet. This latter dependence encodes higher-derivative terms in the supergravity action proportional to the square of the Weyl tensor. Supersymmetry requires that this prepotential be holomorphic and homogeneous of degree two, ${ }^{5}$

$$
F\left(\lambda X^{I}, \lambda^{2} \widehat{A}\right)=\lambda^{2} F\left(X^{I}, \widehat{A}\right) .
$$

Electric-magnetic duality of the theory is realized as symplectic transformations under which the pair $\left(X^{I}, F_{I}\right)$, with $F_{I} \equiv \partial F\left(X^{I}, \hat{A}\right) / \partial X^{I}$, transforms linearly.

The four-dimensional $\mathcal{N}=2$ superconformal algebra is realized as a local gauge symmetry of this theory. As in ordinary gauge theory, one makes a particular choice of gauge in order to perform calculations. The physical observables are, of course, gauge invariant. The superconformal algebra includes a local dilatation invariance under which the vierbein has scaling weight $w=-1$, and the scalars $X^{I}$ have $w=+1$, with associated gauge field $b_{\mu}$, as well as an invariance under special conformal transformations with gauge field $f_{\mu}^{a}$. To gauge-fix the latter, we impose the K-gauge condition $b_{\mu}=0$. To gauge-fixing the former, it is convenient to introduce the symplectically invariant scalar $\mathcal{K}$ via:

$$
e^{-\mathcal{K}}:=-i\left(X^{I} \bar{F}_{I}-\bar{X}^{I} F_{I}\right) .
$$

\footnotetext{
${ }^{4}$ More generally, one can have full-superspace integrals describing higher-derivative interactions. It was shown in [15] that a large class of such terms do not contribute to the quantum entropy. It would be nice to extend this analysis to the level of a complete proof.

${ }^{5}$ The expansion of $F$ in powers of $\widehat{A}$ stands for a derivative expansion in the Lagrangian of the on-shell theory as we discuss in section 6 (see (6.3)).
} 
The field $e^{-\mathcal{K}}$ with scaling weight $w=2$ appears in the action as a conformal compensator, with the kinetic term for the graviton appearing via the combination:

$$
\sqrt{-g} e^{-\mathcal{K}} R \text {. }
$$

The physical, dilatation-invariant metric is $G_{\mu \nu} \equiv e^{-\mathcal{K}} g_{\mu \nu}$.

The local scale invariance is generically gauge-fixed by setting a field with non-zero scaling weight to a constant value. A common choice of gauge is the condition $e^{-\mathcal{K}}=1$ in which we have only $n_{\mathrm{v}}$ fluctuating vector multiplets. In this gauge the original metric $g_{\mu \nu}$ has the standard Einstein-Hilbert Lagrangian for the graviton, as seen easily from the expression (2.3). In this paper we shall use the gauge condition $\sqrt{-g}=1$ which is also very convenient to analyze our problem [12]. In this gauge the fluctuations of the graviton $g_{\mu \nu}$ are constrained to have fixed volume, but we gain a linearly acting symplectic symmetry on the $\left(n_{\mathrm{v}}+1\right)$ freely fluctuating fields $X^{I}$.

We see that one of the $\left(n_{\mathrm{v}}+1\right)$ vector multiplet plays the role of a compensating multiplet. In addition, we need another compensating multiplet to gauge-fix the extra gauge symmetries of the conformal supergravity theory, and we choose this to be a hyper multiplet as in [24]. Unlike the case for vector multiplets, a formalism to treat off-shell $\mathcal{N}=2$ supersymmetry transformations on hyper multiplets with a finite number of auxiliary fields is not known. The compensating hyper multiplet is therefore treated using its equations of motion. We will briefly comment on its consequences in the following subsection.

Conformal $\mathcal{N}=2$ supergravity admits a $\frac{1}{2}$-BPS black hole solution with an $A d S_{2} \times$ $S^{2}$ near-horizon geometry. ${ }^{6}$ The near-horizon solution is fully BPS, as discussed in the introduction. In the gauge $\sqrt{-g}=1$ chosen above, it has the following form (with all other fields not related by symmetries set to zero):

$$
\begin{aligned}
& d s^{2}=\left[-\left(r^{2}-1\right) d t^{2}+\frac{d r^{2}}{r^{2}-1}\right]+\left[d \psi^{2}+\sin ^{2}(\psi) d \phi^{2}\right], \\
& F_{r t}^{I}=e_{*}^{I}, \quad F_{\psi \phi}^{I}=p^{I} \sin \psi, \quad X^{I}=X_{*}^{I}, \quad T_{r t}^{-}=w .
\end{aligned}
$$

Here $F_{\mu \nu}^{I}$ is the field strength of the $\mathrm{U}(1)$ vector field in the vector multiplet $I,\left(e_{*}^{I}, p^{I}\right)$ are real constants and $\left(X_{*}^{I}, w\right)$ are complex constants.

The full-BPS solution (2.4) has a $\mathrm{SL}(2) \times \mathrm{SU}(2)$ bosonic symmetry, the two factors acting on the $A d S_{2}$ and $S^{2}$ parts respectively. It also admits eight supersymmetries, which together with the bosonic symmetries form the $\mathrm{SU}(1,1 \mid 2)$ superalgebra. One of the supercharges that we shall call $Q$ will play an important role in the following. It obeys the algebra

$$
Q^{2}=L_{0}-J_{0}
$$

where $L_{0}$ and $J_{0}$ are the Cartan generators of the $\mathrm{SL}(2)$ and the $\mathrm{SU}(2)$ algebras respectively.

The attractor equations following from full supersymmetry of the near-horizon geometry (or equivalently using the entropy function formalism) are:

$$
e_{*}^{I}-i p^{I}-\frac{w}{2} \bar{X}_{*}^{I}=0, \quad 4 i\left(\bar{w}^{-1} \bar{F}_{I}-w^{-1} F_{I}\right)=q_{I}, \quad|w|^{2}=16 .
$$

\footnotetext{
${ }^{6}$ In this paper we only focus on four-dimensional black holes, but the ideas can clearly be carried forward to higher-dimensional black holes as well. Steps in this directions have been taken in [37].
} 
The phase of the complex number $w$ parametrizing the near-horizon geometry can be set to zero using the $\mathrm{U}(1)_{R}$ gauge symmetry of the theory, which implies $w=4$. This choice also fixes the value of the field $\hat{A}=\left(T_{\mu \nu}^{-}\right)^{2}$ to $\hat{A}=-64$. With this choice, the attractor equations for the scalars are:

$$
X_{*}^{I}+\bar{X}_{*}^{I}=e_{*}^{I} \quad X_{*}^{I}-\bar{X}_{*}^{I}=i p^{I},
$$

and

$$
F_{I}\left(\left(e_{*}^{I}+i p^{I}\right) / 2\right)-\left.\bar{F}_{I}\left(\left(e_{*}^{I}-i p^{I}\right) / 2\right)\right|_{\hat{A}=-64}=i q_{I} .
$$

For such a black hole, using (1.2), the attractor entropy is [38]:

$$
S_{\mathrm{BH}}^{\text {class }}=-\pi q_{I} e_{*}^{I}+\left.4 \pi \operatorname{Im} F\left(\left(e_{*}^{I}+i p^{I}\right) / 2\right)\right|_{\hat{A}=-64} .
$$

At the two-derivative level in the supergravity action, one may recast the above entropy formula in terms of the field $\mathcal{K}$ introduced in (2.2) as follows [39]:

$$
S_{\mathrm{BH}}^{\text {class }}=\pi e^{-\mathcal{K}} .
$$

In this form, it is clear that if we scale all charges as $\left(q_{I}, p^{I}\right) \rightarrow \Lambda\left(q_{I}, p^{I}\right)$ with $\Lambda \rightarrow \infty$, the classical entropy of the black hole scales as $\Lambda^{2}$. We will refer to this scaling behavior later in this paper.

\section{The localization manifold}

In order to apply localization, we must first Wick-rotate the metric and field configuration to Euclidean signature, which is implemented via $t \rightarrow i \tau$ in the metric (2.4) and the field configuration (2.5). All spinors are four-dimensional symplectic MajoranaWeyl spinors [40]. In the conformal supergravity, we have the usual $Q$-supersymmetry transformations and an additional conformal supersymmetry (called $S$-supersymmetry). These transformations are parameterized by the spinors $\xi_{ \pm}^{i}$ and $\eta_{ \pm}^{i}$, respectively. The index $i=1,2$ is an $\mathrm{SU}(2)$ index and \pm denotes the chirality of the spinor. Our conventions are given in appendix A.

The BPS equations of conformal $\mathcal{N}=2$ supergravity are obtained by requiring that the variations of all the fermions in the theory vanish. The vanishing variations of the Weyl multiplet fermions yield the following equations (the details of these equations, including the definitions of the covariant derivative are given in appendix B):

$$
\begin{aligned}
2 D_{\mu} \xi_{ \pm}^{i} \pm \frac{1}{16} T_{a b}^{\mp} \gamma^{a b} \gamma_{\mu} \xi_{\mp}^{i}-\gamma_{\mu} \eta_{\mp}^{i} & =0 \\
\gamma^{\mu} D_{\mu} T_{a b}^{\mp} \gamma^{a b} \xi_{\mp}^{i} \pm 24 D \xi_{ \pm}^{i}-T_{a b}^{\mp} \gamma^{a b} \eta_{ \pm}^{i} & =0 .
\end{aligned}
$$

These equations are known as conformal Killing spinor equations in the literature. The field $D$ that appears in (2.13) is an auxiliary scalar field sitting in the Weyl multiplet. In contrast to (2.12), which determines the Killing spinors of the space-time and thus contains geometrical information, Equation (2.13) does not impose any additional constraints on the geometry and is used to fix the value of the background auxiliary fields $T_{a b}$ and $D$ 
compatible with the conformal Killing spinors. To apply localization, the first step is to find all bosonic backgrounds that admit spinors $\xi_{ \pm}^{i}, \eta_{ \pm}^{i}$ obeying the off-shell BPS equations (2.12), (2.13). This problem was analyzed in [14] by using the equation of motion of the field $D$ at the two-derivative level. Note that the equation of motion can of course change upon including higher-derivative terms [5]. This problem remains to be analyzed with an appropriate off-shell treatment of hyper multiplets. Moreover, it was also assumed in [14] that the $\mathrm{SU}(2)_{R}$ gauge field remains flat on the localization manifold. It is possible that this expectation be confirmed once the gauge field couples to hyper multiplets, but this analysis is beyond our present scope and will not be carried out. The additional onshell input gives a relation between the spinors $\eta_{ \pm}^{i}$ and $\xi_{ \pm}^{i}$, which, in the gauge $e^{-\mathcal{K}}=1$, is simply $\eta_{ \pm}^{i}=0$. This makes it clear that the conformal Killing spinor equations reduce to the usual Killing spinor equations ${ }^{7}$ (generalized to include the $T_{a b}$ auxiliary field of the Weyl multiplet).

With this condition, one can solve the off-shell BPS equations (2.12) with the attractor boundary conditions. The result of [14] is that, in the gauge $\sqrt{-g}=1$, the only solution to these equations ${ }^{8}$ is $A d S_{2} \times S^{2}$. We present the Euclidean metric in a coordinate system that will be useful in the following:

$$
d s^{2}=\sinh ^{2} \eta d \tau^{2}+d \eta^{2}+d \psi^{2}+\sin ^{2} \psi d \phi^{2} .
$$

The coordinate $\eta$ is related to the coordinate $r$ in (2.4) as $r=\cosh \eta$.

To find the complete localization manifold, we have to analyze the off-shell BPS equations $Q \psi_{i}=0$ in the vector multiplets as well. These were analysed in [12, 14], and the result is that the solution set is labelled by one real parameter $C^{I}$ in each vector multiplet:

$$
X^{I}(\eta)=X_{*}^{I}+\frac{C^{I}}{\cosh \eta}, \quad \bar{X}^{I}(\eta)=\bar{X}_{*}^{I}+\frac{C^{I}}{\cosh \eta}, \quad Y_{1}^{I, 1}(\eta)=-Y_{2}^{I, 2}(\eta)=\frac{2 C^{I}}{\cosh ^{2} \eta} .
$$

These scalar field fluctuations actually preserve half of the supersymmetries, they do not obey the equations of motion, and they are supported by the auxiliary fields $Y_{i j}^{I}$ in the vector multiplets.

The final result is that the full localization manifold of the Weyl multiplet coupled to vector multiplets is given by $(2.14),(2.15)$, thus leading to an $\left(n_{\mathrm{v}}+1\right)$-dimensional localization manifold $\mathcal{M}_{Q}$. The coordinates on $\mathcal{M}_{Q}$ used in the formula (1.4) are related to the off-shell fluctuations in (2.15) as:

$$
\phi^{I}=e_{*}^{I}+2 C^{I}=X^{I}(0)+\bar{X}^{I}(0) .
$$

\section{Off-shell supersymmetry transformations and algebra}

We now move to the supersymmetry transformations of the fluctuations around the localizing manifold. The off-shell algebra of our chosen supercharge $Q$ is given to us by the conformal $\mathcal{N}=2$ supergravity formalism - we simply restrict the full off-shell algebra of eight local supercharges to the one supercharge $Q$ that we focus on.

\footnotetext{
${ }^{7}$ See [41] for an analysis of the full off-shell Euclidean conformal Killing spinor equations.

${ }^{8}$ This is true modulo the assumption regarding the $\mathrm{SU}(2)_{R}$ gauge field mentioned above.
} 
Vector multiplets The supersymmetry transformation rules for the vector multiplet using the Killing spinor $\xi_{(1)}^{i}$ given in (B.12) on our localizing background are (from now on, we drop the subscript (1) on the Killing spinor):

$$
\begin{aligned}
Q A_{\mu}^{I} & =\epsilon_{i j}\left(\bar{\xi}_{-}^{i} \gamma_{\mu} \lambda_{+}^{I j}-\bar{\xi}_{+}^{i} \gamma_{\mu} \lambda_{-}^{I j}\right), \\
Q X^{I} & =\epsilon_{i j} \bar{\xi}_{+}^{i} \lambda_{+}^{I j}, \quad Q \bar{X}^{I}=\epsilon_{i j} \bar{\xi}_{-}^{i} \lambda_{-}^{I j}, \\
Q \lambda_{+}^{I i} & =\frac{1}{2} \mathcal{F}_{a b}^{-I} \gamma^{a b} \xi_{+}^{i}+2 \gamma^{\mu} \partial_{\mu} X^{I} \xi_{-}^{i}-Y_{j}^{I}{ }^{j} \xi_{+}^{j}, \\
Q \lambda_{-}^{I i} & =\frac{1}{2} \mathcal{F}_{a b}^{+I} \gamma^{a b} \xi_{-}^{i}-2 \gamma^{\mu} \partial_{\mu} \bar{X}^{I} \xi_{+}^{i}-Y_{j}^{I}{ }^{i} \xi_{-}^{j}, \\
Q Y_{i j}^{I} & =2 \epsilon_{k i} \epsilon_{j l} \bar{\xi}_{+}^{(k} \gamma^{\mu} D_{\mu} \lambda_{-}^{I l)}-2 \epsilon_{k(i} \epsilon_{j) l} \bar{\xi}_{-}^{k} \gamma^{\mu} D_{\mu} \lambda_{+}^{I l},
\end{aligned}
$$

where

$$
\mathcal{F}_{a b}^{-I} \equiv F_{a b}^{-I}-\frac{1}{4} \bar{X}^{I} T_{a b}^{-}, \quad \mathcal{F}_{a b}^{+I} \equiv F_{a b}^{+I}-\frac{1}{4} X^{I} T_{a b}^{+},
$$

and $F_{a b}^{ \pm I}$ is the (anti)self-dual part of the abelian vector field strength $F_{\mu \nu}^{I}=2 \partial_{[\mu} A_{\nu]}^{I}$. The covariant derivative acting on spinors is given by $D_{\mu}=\partial_{\mu}-\frac{1}{4} \omega_{\mu}^{a b} \gamma_{a b}$.

The square of the supersymmetry transformations can be obtained by evaluating the full off-shell algebra [23, 24] on our localizing background (or simply by acting twice with (2.17)):

$$
\begin{aligned}
Q^{2} A_{\mu}^{I} & =i v^{\nu} F_{\nu \mu}^{I}+\partial_{\mu}\left(2 K_{+} \bar{X}^{I}+2 K_{-} X^{I}\right), \\
Q^{2} X^{I} & =i v^{\mu} \partial_{\mu} X^{I}, \quad Q^{2} \bar{X}^{I}=i v^{\mu} \partial_{\mu} \bar{X}^{I}, \\
Q^{2} \lambda_{+}^{I i} & =i v^{\mu} D_{\mu} \lambda_{+}^{I i}+\frac{i}{4} D_{a} v_{b} \gamma^{a b} \lambda_{+}^{I i}, \\
Q^{2} \lambda_{-}^{I i} & =i v^{\mu} D_{\mu} \lambda_{-}^{I i}+\frac{i}{4} D_{a} v_{b} \gamma^{a b} \lambda_{-}^{I i}, \\
Q^{2} Y_{i j}^{I} & =i v^{\mu} \partial_{\mu} Y_{i j}^{I},
\end{aligned}
$$

The transformation parameters in (2.18) are given by

$$
v^{\mu}=-2 i \epsilon_{i j} \bar{\xi}_{+}^{i} \gamma^{\mu} \xi_{-}^{j}, \quad K_{ \pm}=\epsilon_{i j} \bar{\xi}_{ \pm}^{i} \xi_{ \pm}^{j} .
$$

In the right-hand side of (2.18), we use the following useful identities

$$
i D_{[a} v_{b]}=-\frac{1}{4} K_{-} T_{a b}^{-}-\frac{1}{4} K_{+} T_{a b}^{+},
$$

and

$$
\partial_{\mu} K_{ \pm}=\frac{i}{8} v^{\nu} T_{\mu \nu}^{\mp}
$$

which can be derived directly from the definition of the Killing vector and the conformal Killing spinor equations (2.12) with $\eta_{ \pm}^{i}=0$.

Using the explicit form of the Killing spinor (B.12), we find that

$$
v^{\mu}=\left(\begin{array}{llll}
-1 & 0 & 0 & 1
\end{array}\right)^{T},
$$

and

$$
K_{ \pm}=\frac{1}{2}( \pm \cos \psi-\cosh \eta),
$$

which we will use in the next section. 
Hyper multiplets. We consider a set of $n_{H}$ hyper multiplets where the scalars are denoted by $A_{i}{ }^{\alpha}$ with $\alpha=1 \ldots 2 n_{H}$. The index $i$ is a doublet under the $\mathrm{SU}(2) \mathrm{R}$-symmetry, so that we have total of $4 n_{H}$ real scalars. The $4 n_{H}$ fermions are the $2 n_{H}$ positive-chirality spinors $\zeta_{+}^{\alpha}$ and the $2 n_{H}$ negative-chirality spinors $\zeta_{-}^{\alpha}$. We take the hyper multiplet fields to be neutral under the $\mathrm{U}(1)$ gauge symmetry of the vector multiplet, as this is consistent with the classical attractor solution in asymptotically flat space. The scalars $A_{i}{ }^{\alpha}$ span a quaternionic-Kähler manifold and we will assume that the target-space of the hyper multiplet sigma model is flat [42].

Hyper multiplets do not participate in the classical attractor black hole background discussed in section 2 - they take zero or constant values as shown in (2.4), and as a consequence, they do not contribute to the classical action. Their quantum fluctuations, however, are relevant for our discussion, and we will need an off-shell supersymmetry algebra to treat these fluctuations within our approach. For vector multiplets we could directly use the formalism of off-shell conformal supergravity. For hyper multiplets, there is no known off-shell formalism for the full $\mathcal{N}=2$ supersymmetry algebra with a finite number of auxiliary fields.

There is, however, a formalism for the off-shell closure of the algebra of one supercharge for vector and hyper multiplets with a finite number of auxiliary fields [43]. This formalism was used in localization problems in four-dimensional field theory as in $[17,33]$. This algebra acting on vector multiplets is exactly the one given by the conformal $\mathcal{N}=2$ supergravity formalism that we used in the previous section. As was emphasized in [12], the localization solutions (2.15) are universal in the sense that they do not depend on the physical action of the theory and continue to hold even in the presence of other matter fields (which are all constant as in the classical background (2.4)). ${ }^{9}$ We can therefore use the formalism of [43] and $[17,33]$ for hyper multiplets in black hole backgrounds.

The Q-supersymmetry transformation rules are:

$$
\begin{aligned}
Q A_{i}{ }^{\alpha} & =2 \epsilon_{i j}\left(\bar{\xi}_{-}^{j} \zeta_{-}^{\alpha}-\bar{\xi}_{+}^{j} \zeta_{+}^{\alpha}\right), \\
Q \zeta_{+}^{\alpha} & =\gamma^{\mu} \partial_{\mu} A_{i}^{\alpha} \xi_{-}^{i}+2 \epsilon_{i j} \dot{\xi}_{+}^{i} H^{j \alpha}, \\
Q \zeta_{-}^{\alpha} & =\gamma^{\mu} \partial_{\mu} A_{i}^{\alpha} \xi_{+}^{i}+2 \epsilon_{i j} \breve{\xi}_{-}^{i} H^{j \alpha}, \\
Q H^{i \alpha} & =\overline{\breve{\xi}}_{-}^{i} \gamma^{\mu} D_{\mu} \zeta_{+}^{\alpha}-\bar{\xi}_{+}^{i} \gamma^{\mu} D_{\mu} \zeta_{-}^{\alpha},
\end{aligned}
$$

where the action of the covariant derivative on the spinors is exactly as in the vector multiplet. Here, $H^{i \alpha}$ are $4 n_{H}$ scalar auxilary fields. Indeed, upon setting $H^{i \alpha}=0$, one recovers the on-shell transformation rules of [42].

In the off-shell transformations (2.24), the parameters $\breve{\xi}_{ \pm}^{i}$ are built to satisfy:

$$
\begin{aligned}
\bar{\xi}_{-}^{i} \breve{\xi}_{-}^{j} & =\bar{\xi}_{+}^{i} \breve{\xi}_{+}^{j}, \\
\epsilon_{i j} \bar{\xi}_{\mp}^{i} \breve{\xi}_{\mp}^{j} & =\epsilon_{i j} \bar{\xi}_{ \pm}^{i} \xi_{ \pm}^{j}, \\
\epsilon_{i j} \bar{\xi}_{+}^{i} \gamma^{\mu} \breve{\xi}_{-}^{j} & =\epsilon_{i j} \bar{\xi}_{+}^{i} \gamma^{\mu} \xi_{-}^{j} .
\end{aligned}
$$

\footnotetext{
${ }^{9}$ We shall not concern ourselves here with any potentially new solutions to the localization equations in the other matter multiplet sectors. The investigations of [13] and those below indicate that any such solutions will not contribute to the functional integral (1.3), but we cannot prove this at the moment.
} 
In these equations, the spinors $\xi_{ \pm}^{i}$ are given by (B.12) as before. As mentioned in [33], the constraints $(2.25)$ do admit non-trivial solutions to $\breve{\xi}_{ \pm}^{j}$, and we present an explicit solution for our background in appendix C. With these constraints, the Q-supersymmetry transformations close off-shell:

$$
\begin{aligned}
Q^{2}{A_{i}}^{\alpha} & =i v^{\mu} \partial_{\mu} A_{i}{ }^{\alpha}, \\
Q^{2} \zeta_{+}^{\alpha} & =i v^{\mu} D_{\mu} \zeta_{+}^{\alpha}+\frac{i}{4} D_{a} v_{b} \gamma^{a b} \zeta_{+}^{\alpha}, \\
Q^{2} \zeta_{-}^{\alpha} & =i v^{\mu} D_{\mu} \zeta_{-}^{\alpha}+\frac{i}{4} D_{a} v_{b} \gamma^{a b} \zeta_{-}^{\alpha}, \\
Q^{2} H^{i \alpha} & =i v^{\mu} \partial_{\mu} H^{i \alpha} .
\end{aligned}
$$

For the localization analysis, we set all the fermion variations under $Q$ in (2.24) to zero. It is clear that the configuration where the auxiliary field $H^{i \alpha}=0$ and the hyper multiplet scalars $A_{i}^{\alpha}=$ constant is a solution to the above BPS equations. In order to find an exhaustive list of all solutions, one needs to do an analysis as in [14] by separating the different tensor structures on the right-hand side. For now, we proceed with the trivial solutions.

Supersymmetry algebra of $\boldsymbol{Q}$. Inspection of (2.18) and (2.26) shows that supersymmetry algebra of $Q$ acting on all fields of the vector and hyper multiplets in the $A d S_{2} \times S^{2}$ background is:

$$
Q^{2}=i \delta_{\text {cgct }}(v)+i \delta_{M}\left(L_{a b}\right)+\delta_{\text {gauge }}\left(\theta^{I}\right),
$$

where the quantities on the right-hand side are as follows. The operator $\delta_{\text {cgct }}(v)$ is the covariant general coordinate transformation, defined in e.g. [24], which is the variation under all gauge symmetries of the conformal supergravity theory (including regular general coordinate transformations, but also e.g. the U(1) gauge symmetry of the vector multiplets), with the gauge parameters determined by the vector $v^{\mu}$ (given by (2.19) for our background). In our case, it is equal to the sum of the Lie derivative along the vector $v^{\mu}$ and the $\mathrm{U}(1)$ gauge transformation parametrized by $-v^{\mu} A_{\mu}^{I}$. The transformation $\delta_{M}$ is a Lorentz transformation parametrized by (see (2.20))

$$
L_{a b}:=\frac{i}{4}\left(K_{+} T_{a b}^{+}+K_{-} T_{a b}^{-}\right)=D_{[a} v_{b]},
$$

which, on our background solution, equals

$$
L_{a b}=\left(\begin{array}{cccc}
0 & \cosh \eta & 0 & 0 \\
-\cosh \eta & 0 & 0 & 0 \\
0 & 0 & 0 & \cos \psi \\
0 & 0 & -\cos \psi & 0
\end{array}\right) .
$$

Lastly, the transformation $\delta_{\text {gauge }}$ is a $\mathrm{U}(1)$ gauge transformation parametrized by

$$
\theta^{I}:=2 K_{+} \bar{X}^{I}+2 K_{-} X^{I} .
$$

In the following, we will combine the off-shell supersymmetry $Q$ with the BRST symmetry encoding the $\mathrm{U}(1)$ gauge symmetry of the vector multiplet. To do so, we isolate 
the $\mathrm{U}(1)$ gauge connection term present in the covariant general coordinate transformation of (2.27) and combine it with the gauge transformation already present in the algebra of $Q$. We thus rewrite the off-shell supersymmetry algebra as ${ }^{10}$

$$
Q^{2}=i \mathcal{L}_{v}+i \delta_{M}\left(L_{a b}\right)+\delta_{\text {gauge }}\left(\widehat{\theta}^{I}\right)
$$

where $\mathcal{L}_{v}$ is the Lie derivative along the vector $v$, and

$$
\widehat{\theta}^{I}:=2 K_{+} \bar{X}^{I}+2 K_{-} X^{I}-i v^{\mu} A_{\mu}^{I} .
$$

Using the values (2.5) of the background gauge fields $A_{\mu}^{I}$ on the localizing manifold, we obtain the explicit expression:

$$
\widehat{\theta}^{I}=-e_{*}^{I}-2 C^{I}=-\phi^{I}
$$

Note that the gauge parameters on the right-hand side of the supersymmetry algebra are precisely the coordinates on the localizing manifold.

We note that the algebra (2.31) of the supercharge $Q$ is similar in structure, but not quite the same, as the one appearing in $[17,33]$. Before specifying the background manifold, the off-shell supersymmetry transformations (2.17), (2.24) are the same as the corresponding ones in $[17,33]$. The reason for the difference is simply that the background values of all the supergravity fields are different. In particular, the right-hand side of the algebra (2.31) involves the SU(2) R-symmetry of supergravity in the case of the sphere, while this term is absent in our case. Instead, the $A d S_{2} \times S^{2}$ algebra contains a Lorentz rotation which the sphere algebra does not have. This fact will play a role in our analysis of the index theorem in section 4 .

\section{Gauge-fixing and the introduction of ghosts}

We now turn to the issue of gauge-fixing the U(1) symmetry in each vector multiplet. The main problem is that the action of fixing a gauge does not commute with the off-shell supersymmetry - which is central to our localization methods. To treat this problem, we will need to extend the off-shell supersymmetry algebra of $Q$ to include the effect of the gauge-fixing. We also saw a hint of this appearing in the fact that the supercharge $Q$ squares to a compact bosonic generator only modulo a gauge transformation in Equation (2.31).

It is natural to solve this problem by combining the conformal $\mathcal{N}=2$ supergravity formalism with the covariant BRST formalism ${ }^{11}$ by adding Fadeev-Popov ghosts to the

\footnotetext{
${ }^{10}$ We note here that a similar procedure can be used to combine the spin-connection term appearing in the covariant general coordinate transformation of fermions with the Lorentz transformation parameter $L_{a b}$. In the Lorentz gauge where $\omega_{\tau}^{12}=-\cosh \eta, \omega_{\phi}^{34}=\cos \psi$, this yields $\widehat{L}_{a b}:=L_{a b}-v^{\mu} \omega_{\mu}^{a b}=0$, so that the supersymmetry algebra is simply $Q^{2}=i v^{\mu} \partial_{\mu}+\delta_{\text {gauge }}\left(\widehat{\theta}^{I}\right)$. In this paper, we will stay in a generic Lorentz gauge where such cancellations need not happen.

${ }^{11}$ Another, more hands-on method is to choose a suitable gauge-fixed background and to compute the bosonic and fermionic eigenmodes around this background. The non-cancellation then happens because the naive $Q$ operator, upon acting on a certain eigenmode, moves us out of the gauge-fixing condition and one therefore has to modify $Q$ as in e.g. [44].
} 
theory. The technical task is to set up a BRST complex for the gauge symmetries of the theory, and combine it with the off-shell supersymmetry complex generated by $Q$. This procedure builds a new supercharge $\widehat{Q}$ which, as we will demonstrate, is suitable for localization and encodes both the gauge symmetry and the supersymmetry of the action. Once this formalism has been set up, the approach turns out to be extremely compact, and we can use index theory to elegantly compute the required functional determinants as laid out in [17].

To treat the $\mathrm{U}(1)$ gauge symmetry of the vector multiplet, we introduce a standard BRST ghost system. A U(1) gauge transformation acts on the vector fields as

$$
\delta_{g} A_{\mu}^{I}=\partial_{\mu} \lambda^{I}
$$

where $\lambda^{I}$ is the parameter of the transformation in each vector multiplet. To each of these transformations we associate a ghost $c^{I}$ along with an anti-ghost $b^{I}$ and a Lagrange multiplier $B^{I}$. Notice that the operator $\partial_{\mu}$ has normalizable zero modes on a compact space, namely any constant function. In order to treat these zero modes we need to introduce the so-called ghost-for-ghosts: the constant field $c_{0}^{I}$, along with two BRST-trivial pairs $\left(\bar{\eta}^{I}, B_{1}^{I}\right)$ and $\left(\eta^{I}, \bar{B}_{1}^{I}\right)$. This is the required field content to properly fix the gauge in the path integral (1.4). This fact is most easily understood by making use of the BatalinVilkovisky formalism [31, 45] and noting that the gauge theory at hand is a first stage reducible theory.

The BRST transformation laws of the vector multiplet fields in the adjoint of the U(1) gauge group are:

$$
\delta_{B} A_{\mu}^{I}=\Lambda \partial_{\mu} c^{I}, \quad \delta_{B} X^{I}=0, \quad \delta_{B} \bar{X}^{I}=0, \quad \delta_{B} \lambda_{+}^{i I}=0, \quad \delta_{B} \lambda_{-}^{i I}=0, \quad \delta_{B} Y_{i j}^{I}=0,
$$

with $\Lambda$ a constant anti-commuting parameter parametrizing the BRST transformation. We also have the following transformations on the ghost fields:

$$
\delta_{B} b^{I}=\Lambda B^{I}, \quad \delta_{B} B^{I}=0, \quad \delta_{B} \eta^{I}=\Lambda \bar{B}_{1}^{I}, \quad \delta_{B} \bar{B}_{1}^{I}=0, \quad \delta_{B} \bar{\eta}^{I}=\Lambda B_{1}^{I}, \quad \delta_{B} B_{1}^{I}=0,
$$

and

$$
\delta_{B} c^{I}=\Lambda c_{0}^{I}, \quad \delta_{B} c_{0}^{I}=0 .
$$

The operator $Q_{B}$ defined by $\delta_{B} \phi:=\Lambda Q_{B} \phi$ ( $\phi$ being any field of the theory) is a nilpotent operator, due to the fact that the field $c_{0}^{I}$ is constant.

We now add to the $\mathcal{N}=2$ supergravity Lagrangian a $Q_{B}$-exact gauge-fixing term:

$$
\mathcal{L}_{\text {g.f. }}=Q_{B}\left[b^{I}\left(-\frac{B^{I}}{2 \xi_{A}}+G^{A}\left(A_{\mu}^{I}\right)\right)+\bar{\eta}^{I}\left(-\frac{B_{1}^{I}}{2 \xi_{c}}+G^{c}\left(c^{I}\right)\right)+\eta^{I}\left(-\frac{\bar{B}_{1}^{I}}{2 \xi_{b}}+G^{b}\left(b^{I}\right)\right)\right],
$$

where $G^{A}, G^{c}$ and $G^{b}$ are appropriate gauge-fixing functions for the vector field, the ghost and the anti-ghost, respectively, and $\xi_{A}, \xi_{b}$ and $\xi_{c}$ are constant parameters. The gauge-fixed action

$$
S_{\text {gauge-fixed }}=S_{0}+\int d^{4} x \mathcal{L}_{\text {g.f. }},
$$


where $S_{0}$ is the action of vector and hyper multiplets coupled to conformal supergravity, is BRST invariant since $\mathcal{L}_{\text {g.f. }}$ is $Q_{B}$-exact and $Q_{B}$ is nilpotent. Expanding (3.5) using the BRST transformation rules leads to the expression

$$
\begin{aligned}
S_{\text {g.f. }}=\int d^{4} x \mathcal{L}_{\text {g.f. }} & \\
=\int d^{4} x & \left\{B^{I}\left(G^{A}\left(A_{\mu}^{I}\right)-\frac{B^{I}}{2 \xi_{A}}-\eta^{I} \frac{\delta G^{b}}{\delta b^{I}}\right)-b^{I} \frac{\delta G^{A}\left(A_{\mu}^{I}\right)}{\delta A_{\mu}^{J}} \partial_{\mu} c^{J}\right. \\
& \left.+\bar{B}_{1}^{I}\left(G^{b}\left(b^{I}\right)-\frac{\bar{B}_{1}^{I}}{2 \xi_{b}}\right)+B_{1}^{I}\left(G^{c}\left(c^{I}\right)-\frac{B_{1}^{I}}{2 \xi_{c}}\right)-c_{0}^{I} \bar{\eta}^{J} \frac{\delta G^{c}\left(c^{I}\right)}{\delta c^{J}}\right\} .
\end{aligned}
$$

One can recognize in this action the field $B^{I}$ as a Gaussian-weighted Lagrange multiplier for the gauge condition $G^{A}\left(A_{\mu}^{I}\right)=\eta^{J} \frac{\delta G^{b}\left(b^{I}\right)}{\delta b^{J}}$, the field $B_{1}^{I}$ as a Gaussian-weighted Lagrange multiplier for the gauge condition $G^{c}\left(c^{I}\right)=0$ and the field $\bar{B}_{1}^{I}$ as a Gaussianweighted Lagrange multiplier for the gauge condition $G^{b}\left(b^{I}\right)=0$. For the case at hand, these last two gauge-fixing functions are supposed to freeze the freedom one has in shifting the ghost and anti-ghost by a constant function, and we can thus take them specifically to be $G^{c}\left(c^{I}\right)=c^{I}$ and $G^{b}\left(b^{I}\right)=b^{I}$. The $B_{1}^{I}, \bar{B}_{1}^{I}$ Lagrange multipliers then impose the conditions that $\int c^{I}=0$ and $\int b^{I}=0$, respectively. The gauge-fixing function for the gauge field $A_{\mu}^{I}$ is then fixed to $G^{A}\left(A_{\mu}^{I}\right)=\eta^{I}$ through the equation of motion for the Lagrange multiplier $B^{I}$. Note also that the partition function computed from this gauge-fixed action is independent of the $\xi_{A}, \xi_{c}$ and $\xi_{b}$ parameters [17].

We pause here for a moment in order to make a technical comment on the ghost set up that was used in [17]. For non-abelian gauge theories, like the one considered in [17], constant functions like $c_{0}$ are not zero modes of the operator $D_{\mu}^{a}$ (where $a$ is a color index). One could have tried to set up the ghost-for-ghost $c_{0}$ to be a zero mode of the covariant derivative and thus take it to be a covariantly constant function - indeed, this may seem natural from a certain point of view. Doing so, however, would render the integrations over the gauge field and the ghost-for-ghost inter-dependent inside the path-integral, which is difficult to implement in practice. The strategy for non-abelian gauge fields considered in [17] was to keep $c_{0}$ as a constant function, and use a BRST charge which is non-nilpotent. In our case the gauge symmetry is abelian.

We now apply the above formalism to our problem of abelian vector multiplets on $A d S_{2} \times S^{2}$. The non-compact nature of the space introduces some subtleties. Firstly, we need to specify boundary conditions on all the fields. For the physical fields, we choose boundary conditions as in $[26,46]$. For the ghost fields, we impose Dirichlet boundary conditions on the fields $b^{I}, c^{I}$. This implies that there is no normalizable zero modes for these fields, and therefore no ghost-for-ghosts. This is consistent with the boundary conditions used in [47] for the gauge parameters. Using this formalism, we set all the ghost-for-ghost fields to zero hereafter.

Secondly, there is the issue of boundary modes which are normalizable modes of the gauge fields $A_{\mu}^{I}$ that are formally pure gauge, but with gauge parameters that do not vanish at infinity (these have been called "discrete modes" [47]). The four-dimensional bulk action 
depends only on gauge invariant quantities and therefore does not depend on these discrete modes - thus naively giving a divergence in the path integral. These special modes have been treated carefully in [47], and the idea is to obtain their contribution separately using arguments of ultra-locality. This gives rise to a factor of $\ell^{-2 \beta}$ to the functional integral, where $\ell$ is the background length scale of the problem and $\beta$ depends on the field under consideration. The non-zero modes can be treated as usual, but since we need a complete set of local fields in the computation, we should add and subtract one set of zero modes ${ }^{12}$ to the non-zero modes, thus obtaining the contribution of a complete local set of modes and a factor of $\ell^{2}$. As a result, we need to multiply the answer found by using a complete set of local field observables by a factor $\ell^{2-2 \beta}$. For the gauge fields, one has $\beta=1$ [47], which effectively means that the discrete modes do not contribute to the determinant calculation. ${ }^{13}$

The combined supercharge $\widehat{Q}$ and its algebra. We now consider the combined transformation for the BRST symmetry and the off-shell supersymmetry, generated by $\widehat{Q} \equiv$ $Q+Q_{B}$. We require this new supercharge to square to

$$
\widehat{Q}^{2}=i \mathcal{L}_{v}+i \delta_{M}\left(L_{a b}\right) \equiv H
$$

where $\mathcal{L}_{v}$ and $\delta_{M}$ are the Lie derivative and the Lorentz transformations defined around Equation (2.31). Note that the vector multiplet gauge transformation is no longer present on the right-hand side of the algebra (3.8) - precisely because it is already encoded in the BRST symmetry. The above algebra (3.8) allows us to systematically derive the supersymmetry transformation rules on the ghost system. Expanding $\widehat{Q}^{2}$, and using the algebra (2.31) for $Q$ and the nilpotency of $Q_{B}$, we obtain

$$
\widehat{Q}^{2}=Q^{2}+Q_{B}^{2}+\left\{Q, Q_{B}\right\}=i \mathcal{L}_{v}+i \delta_{M}\left(L_{a b}\right)+\delta_{\text {gauge }}\left(\widehat{\theta}^{I}\right)+\left\{Q, Q_{B}\right\}
$$

Comparing with (3.8), we deduce that the anticommutator of a supersymmetry and a BRST transformation on the physical and auxiliary fields of the theory should compensate for the gauge transformation parametrized by the vector and scalar fields of the vector multiplet. Applying this observation to the various fields leads to the supersymmetry transformation rules for the ghost system.

As an example, consider the vector field $A_{\mu}^{I}$ :

$$
\left\{Q, Q_{B}\right\} A_{\mu}^{I}=Q\left(\partial_{\mu} c^{I}\right)=-\partial_{\mu}\left(\widehat{\theta}^{I}\right),
$$

which immediately yields

$$
Q c^{I}=-\widehat{\theta}^{I}
$$

\footnotetext{
${ }^{12}$ In order to justify this procedure more carefully in our localization computation, one needs to analyze the cut-off theory and carefully take an infinite-volume limit. This must be done in such a way as to keep the local superalgebra and the completeness of the basis intact. Another possible resolution of this subtlety is that boundary effects will lift these zero modes in the localization action, as consistent with the fact that $H$ takes non-zero values on these modes. The boundary conditions introduced in the context of the $A d S / C F T$ in [48] may be relevant to this discussion.

${ }^{13}$ In contrast, these modes are expected to play a role in the graviton calculation.
} 
Applying $\widehat{Q}^{2}$ to the other fields of the theory, we obtain the remaining supersymmetry transformations ${ }^{14}$

$$
Q b^{I}=0, \quad Q B^{I}=i \mathcal{L}_{v} b^{I} .
$$

We can now write down the various anticommutators on all fields of the theory as

$$
\begin{aligned}
Q^{2} \Phi^{\left({ }^{\prime}\right)} & =\left(i \mathcal{L}_{v}+i \delta_{M}\left(L_{a b}\right)+\delta_{\text {gauge }}\left(\widehat{\theta}^{I}\right)\right) \Phi^{\left({ }^{\prime}\right)}, & \left.Q^{2} \text { (gh. }\right) & =0, \\
Q_{B}^{2} \Phi^{\left({ }^{\prime}\right)} & =0, & \left.Q_{B}^{2} \text { (gh. }\right) & =0, \\
\left\{Q, Q_{B}\right\} \Phi^{\left({ }^{\prime}\right)} & =-\delta_{\text {gauge }}\left(\widehat{\theta}^{I}\right) \Phi^{\left({ }^{\prime}\right)}, & \left.\left\{Q, Q_{B}\right\} \text { (gh. }\right) & =i \mathcal{L}_{v} \text { (gh.) },
\end{aligned}
$$

where $\Phi^{\left({ }^{\prime}\right)}$ stands for bosonic (fermionic) physical and auxiliary fields, and gh. stands for all the ghost field of the gauge-fixing complex. Using these transformation rules, we conclude that the complete set of fields (including the ghosts) now admits a symmetry $\widehat{Q}$ realized off-shell with algebra (3.8). This is the supercharge that we would like to use to perform localization, and the localizing arguments need to be reapplied with this new operator.

The first observation to be made is that the complete gauge-fixed action is closed under $\widehat{Q}$,

$$
\widehat{Q}\left(S_{0}+S_{\text {g.f. }}\right)=0 .
$$

This is the case since the $S_{0}$ action is gauge and supersymmetry invariant by definition, and as was established in [17], one may replace $Q_{B}$ in (3.5) by $\widehat{Q}$ without changing the value of the path integral under consideration. Thus, the gauge-fixed action we built by introducing the gauge-fixing complex is closed under the $\widehat{Q}$ operator, and this operator squares to a sum of bosonic symmetries. This is the correct setup for localization.

We also need to revisit the conditions for the saddle point around which the localization is performed. This means we now look for solutions to the equation

$$
\widehat{Q} \psi_{i}=Q \psi_{i}+Q_{B} \psi_{i}=0
$$

for all physical fermions $\psi_{i}$ in the theory. For the gaugini in the adjoint representation of the gauge group, the added term $Q_{B} \lambda_{ \pm}^{I^{i}}$ is zero and therefore does not modify the initial solution found for $Q \lambda=0$ in [12]. A similar statement can be made for the fermions of the hyper multiplets.

Finally, we need to modify the deformation operator $Q \mathcal{V}$ used in localization to the operator $\widehat{Q} \widehat{\mathcal{V}}$ which now includes the gauge-fixing part of the action (3.5):

$$
\widehat{\mathcal{V}} \equiv \mathcal{V}+\mathcal{V}_{\text {g.f. }}=\int d^{4} x\left[\sum_{i}\left(Q \psi_{i}, \psi_{i}\right)+b^{I} G^{A}\left(A_{\mu}^{I}\right)\right],
$$

where, following the discussion below Equation (3.7), we have discarded the ghost-for-ghost fields and taken the parameter $\xi_{A}$ to infinity in the gauge-fixing action. Here we point out that the Euclidean analytic continuation of the spinors that we chose in section 2 is not compatible with the positive-definiteness of the action $\widehat{Q} \widehat{\mathcal{V}}$. So, one has to make a choice

\footnotetext{
${ }^{14}$ The same procedure can be applied to also determine the transformation rules for the ghost-for-ghost fields when they are present, e.g. as in [17].
} 
between supersymmetry and positive-definiteness. We choose to preserve supersymmetry, and as we see in the next section, we obtain a sensible final result. We take this to mean that for the unpaired modes under $(-1)^{F}$ (that is, for the index computation), the fluctuation determinant is well-defined. The other choice of analytic continuation includes its own complications (e.g. new localizing solutions), as discussed in [36].

We now have the full formalism in place to compute the super-determinant of the $\widehat{Q} \widehat{\mathcal{V}}$ operator over the $\widehat{Q}$-complex (2.17), (3.2), (2.24), which we proceed to do.

\section{Calculation of the one-loop determinant}

In this section we compute the one-loop determinant of the $\widehat{Q} \widehat{\mathcal{V}}$ operator using an index theorem. We follow the procedure as explained in $[17,19,33,49] .{ }^{15}$ We will first organize the various fields on which the $\widehat{Q}$ operator acts in bosonic and fermionic quantities as:

$$
X^{a} \stackrel{\widehat{Q}}{\rightarrow} \widehat{Q} X^{a}, \quad \Psi^{\alpha} \stackrel{\widehat{Q}}{\rightarrow} \widehat{Q} \Psi^{\alpha}
$$

where $X^{a}$ and $\Psi^{\alpha}$ stand for fundamental bosons and fermions, respectively. The full set of bosonic and fermionic fields of the theory are thus organized as:

$$
\mathfrak{B} \equiv\left\{X^{a}, \widehat{Q} \Psi^{\alpha}\right\} \text { (bosonic), } \quad \mathfrak{F} \equiv\left\{\Psi^{\alpha}, \widehat{Q} X^{a}\right\} \text { (fermionic) }
$$

The field-splittings for the vector and hyper multiplets are shown in appendix C. With this change of variables, the deformation operator $\widehat{\mathcal{V}}=\mathcal{V}+\mathcal{V}_{\text {gf }}$ can be written, up to quadratic order in the fields, as follows:

$$
\left.\widehat{\mathcal{V}}\right|_{\text {quad. }}=(\widehat{Q} X \Psi)\left(\begin{array}{cc}
D_{00} & D_{01} \\
D_{10} & D_{11}
\end{array}\right)\left(\begin{array}{c}
X \\
\widehat{Q} \Psi
\end{array}\right) .
$$

This implies the following form for $\widehat{Q} \widehat{\mathcal{V}}$ :

$$
\begin{aligned}
\left.\widehat{Q} \widehat{\mathcal{V}}\right|_{\text {quad. }} & =\int d^{4} x\left(\mathfrak{B} K_{b} \mathfrak{B}+\mathfrak{F} K_{f} \mathfrak{F}\right) \equiv \mathcal{L}_{b}+\mathcal{L}_{f}, \\
\mathcal{L}_{b} & =(X \widehat{Q} \Psi)\left(\begin{array}{cc}
H & 0 \\
0 & 1
\end{array}\right)\left(\begin{array}{cc}
D_{00} & D_{01} \\
D_{10} & D_{11}
\end{array}\right)\left(\begin{array}{c}
X \\
\widehat{Q} \Psi
\end{array}\right)
\end{aligned}
$$

and

$$
\mathcal{L}_{f}=(\widehat{Q} X \Psi)\left(\begin{array}{cc}
D_{00} & D_{01} \\
D_{10} & D_{11}
\end{array}\right)\left(\begin{array}{cc}
1 & 0 \\
0 & H
\end{array}\right)\left(\begin{array}{c}
\widehat{Q} X \\
\Psi
\end{array}\right)
$$

and where $H=\widehat{Q}^{2}$ as defined in (3.8).

By definition, the one-loop determinant for the operator $\widehat{Q} \widehat{\mathcal{V}}$ is:

$$
Z_{1-\text { loop }}=\left(\frac{\operatorname{det} K_{f}}{\operatorname{det} K_{b}}\right)^{\frac{1}{2}}
$$

\footnotetext{
${ }^{15}$ We thank Sungjay Lee for many informative discussions about this topic.
} 
From equations (4.4), (4.5), (4.6), we have that

$$
\frac{\operatorname{det} K_{f}}{\operatorname{det} K_{b}}=\frac{\operatorname{det}_{\Psi} H}{\operatorname{det}_{X} H}=\frac{\operatorname{det}_{\operatorname{Coker} D_{10} H}}{\operatorname{det}_{K e r D_{10}} H} .
$$

The above ratio of determinants can be computed from the knowledge of the index

$$
\operatorname{ind}\left(D_{10}\right)(t):=\operatorname{Tr}_{\operatorname{Ker} D_{10}} e^{-i H t}-\operatorname{Tr}_{\operatorname{Coker} D_{10}} e^{-i H t} .
$$

Indeed the expansion of the index

$$
\operatorname{ind}\left(D_{10}\right)(t)=\sum_{n} a(n) e^{-i \lambda_{n} t},
$$

encodes the eigenvalues $\lambda_{n}$ of $H$, as well as their indexed degeneracies $a(n)$, and we can thus write the ratio of determinants in (4.8) as:

$$
\frac{\operatorname{det}_{\mathrm{Coker} D_{10}} H}{\operatorname{det}_{\operatorname{Ker} D_{10}} H}=\prod_{n} \lambda_{n}^{-a(n)} .
$$

This infinite product is a formal expression, and we will discuss a suitable regulator in the following.

From a mathematical point of view, the index (4.9) is an equivariant index with respect to the action of $H$. This operator acts on all the fields as $H=i \mathcal{L}_{v}+i \delta_{M}\left(L_{a b}\right)$ according to (3.8). The action of $H$ on the spacetime manifold is simply through the Lie derivative, i.e. the $\mathrm{U}(1)$ action $H=\left(-i \partial_{\tau}+i \partial_{\phi}\right) \equiv L_{0}-J_{0}$. A U(1)-equivariant index of this type can be computed in an elegant manner using the Atiyah-Bott index theorem for transversally elliptic operators [50], as explained in detail in [17]. Here we will make use of this index theorem even though $A d S_{2}$ is a non-compact space. We note in this context that the AdS space is effectively compact, in the sense that there is a gravitational potential well that localizes physical excitations around the fixed point of the $\mathrm{U}(1)$ action. This suggests that continuous modes do not contribute to the index, which is what we will assume. We leave a detailed analysis of the boundary conditions and boundary action as an interesting problem to be analyzed in the future. We summarize the ideas of the index theorem very briefly from a working point of view in appendix $\mathrm{C}$, where we also show that the $D_{10}$ operator is transversally elliptic with respect to the action of $H$. The result of the theorem applied to our problem is that the index of the $D_{10}$ operator (4.9) reduces to the fixed points of the manifold under the action of $H$. Denoting this action by $x \mapsto \widetilde{x}=e^{-i H t} x$, we have:

$$
\operatorname{ind}\left(D_{10}\right)(t)=\sum_{\{x \mid \widetilde{x}=x\}} \frac{\operatorname{Tr}_{X, \Psi}(-1)^{F} e^{-i H t}}{\operatorname{det}(1-\partial \widetilde{x} / \partial x)} .
$$

In our case the action of $H$ on $A d S_{2} \times S^{2}$ decomposes into the separate actions of $L_{0}$ and $J_{0}$ on the $A d S_{2}$ and $S^{2}$ factors, respectively. There are two fixed points - at the center $\eta=0$ of the $A d S_{2}$ factor (fixed by the rotation $L_{0}$ ), and at the two poles on the $S^{2}$ factor (fixed by the rotation $J_{0}$ ). To apply the index theorem, we further need to know the explicit field content of $X$ and $\Psi$, and the charges they carry under $H$. Once we know the 
eigenvalues of all the fields under $H$, we can compute the trace in the numerator of (4.12). As we discussed in section 2, the off-shell algebra that we use has the same structure as that used in $[17,33]$, in that the field content and the gauge invariances are the same. This allows us to use the splitting of fields into $X, \Psi$ as used by those authors. On the other hand, as was emphasized at the end of section 2, the physical transformations on the right-hand side of the algebra as well as the background manifold are different, and we should use the algebra (3.8) that is relevant for our problem here.

The action of the Lie derivative $\mathcal{L}_{v}$ on any field of the theory is composed of two parts: a local translation on the spacetime coordinates along the vector $v^{\mu}$, and an action on the tensor indices of the field. At the fixed points of spacetime under $H$, the former action vanishes by definition. Thus, in order to compute the action of $H$, we only need to keep track of the latter action of the Lie derivative, as well as the action of the Lorentz rotation $L_{a b}$. The vector $v^{\mu}(2.22)$ translates us along the angles $\tau$ and $\phi$ in the metric (2.14) and is therefore essentially a rotation around the fixed points. The operator $L_{a b}(2.29)$ at the fixed points is also the same rotation (acting on the spin part of the fields). Therefore, we only need to compute the charges of the all fields under a rotation around the center of $A d S_{2}$ combined with a rotation around $S^{2}$.

The calculation is simplified by going to complex coordinates in which the $A d S_{2} \times S^{2}$ metric is

$$
d s^{2}=\ell^{2}\left(\frac{4 d w d \bar{w}}{(1-w \bar{w})^{2}}+\frac{4 d z d \bar{z}}{(1+z \bar{z})^{2}}\right) .
$$

Here $\ell$ is the overall physical size of the $A d S_{2} \times S^{2}$ metric, which is governed by the fielddependent physical metric $e^{-\mathcal{K}\left(X^{I}\right)} g_{\mu \nu}$ that depends on the position in the $A d S_{2}$ space. At the fixed points, i.e. the center of $A d S_{2}$, this size is given by $\ell^{2}=e^{-\mathcal{K}\left(\phi^{I}\right)}$ in the gauge $\sqrt{-g}=1 .{ }^{16}$ At the fixed points, we have $w=0$, and $z=0$ or $1 / z=0$. There, the action of the operator $e^{-i H t}$ on the spacetime coordinates is $(z, w) \rightarrow e^{-i t / \ell}(z, w)$. Therefore, the determinant factor in the denominator of $(4.12)$ is $(1-q)^{2}\left(1-q^{-1}\right)^{2}$ with $q=e^{-i t / \ell}$.

Near the fixed points the space looks locally like $\mathbb{R}^{4}$ with an associated $\mathrm{SO}(4)=$ $\mathrm{SU}(2)_{+} \times \mathrm{SU}(2)_{-}$rotation symmetry. The planes labelled by the two complex coordinates $(z, w)$ rotate in the same direction under the $\mathrm{SU}(2)_{+}$, and in opposite directions under $\mathrm{SU}(2)_{-}$. Comparing the two forms of the metric (2.4) and (4.13) (noting the change in orientiation of the $S^{2}$ part when going from one to another), and recalling that $H=-i \partial_{\tau}+i \partial_{\phi}$, we identify the action of $H$ with the Cartan generator of $\mathrm{SU}(2)_{+}$at the North Pole, and with the Cartan of $\mathrm{SU}(2)_{-}$at the South Pole according to:

$$
H=2 J_{+} \quad(\mathrm{NP}), \quad H=2 J_{-} \quad(\mathrm{SP}) .
$$

We now need to compute the charges of all the fields under this generator.

Vector multiplets. In the vector multiplet sector, the fields are separated into $X=$ $\left\{X^{I}-\bar{X}^{I}, A_{\mu}^{I}\right\}$ and $\Psi=\left\{\Xi^{I i j}, c^{I}, b^{I}\right\}$, and their images under $\widehat{Q}$. We discuss some more details of this splitting in appendix C. The fermions $\Xi^{I i j}$ are defined as

$$
\Xi^{I i j}:=2 \bar{\xi}_{+}^{(i} \lambda_{+}^{I j)}+2 \bar{\xi}_{-}^{(i} \lambda_{-}^{I j)} .
$$

\footnotetext{
${ }^{16}$ Here and in the following, we write $\mathcal{K}\left(\phi^{I}\right)$ to mean $\mathcal{K}\left(\phi^{I}+i p^{I}\right)$.
} 
The scalars $\left(X^{I}-\bar{X}^{I}\right), c^{I}, b^{I}$ are in the $(\mathbf{0}, \mathbf{0})$ of $\mathrm{SO}(4)=\mathrm{SU}(2)_{+} \times \mathrm{SU}(2)_{-}$, and therefore are uncharged under $H$. The vector rotates with spin one, and therefore is in the $(\mathbf{2}, \mathbf{2})$ of the $\mathrm{SO}(4)$. There are two modes $\left(A_{z}, A_{w}\right)$ with charges +1 and two modes $\left(A_{\bar{z}}\right.$, $\left.A_{\bar{w}}\right)$ with charges -1 under $H .{ }^{17}$ To compute the charges of the spinor bilinears, we notice that the spinor $\xi_{+}$vanishes at the north pole, and so the bilinear $\Xi^{I i j}$ is in the $(\mathbf{1}, \mathbf{3})$ of the $\mathrm{SO}(4)$. The spinor bilinears $\Xi^{I}{ }^{i j}$ thus carry charge 0 under $H$. Similarly, at the south pole, the spinor bilinears are in the $(\mathbf{3}, \mathbf{1})$, while $H$ is the Cartan of the $\mathrm{SU}(2)_{-}$. All this is consistent with the explicit symbol computation in appendix $\mathrm{C}$, where the coupling of the bilinears with the self-dual and the anti-self-dual part of the field strength is computed.

Putting all this together, we find that, at each of the poles, the contribution to the index is:

$$
\left[\frac{2 q}{(1-q)^{2}}\right]
$$

We see that there is a pole in this expression when $q=1$. This pole arises due to the fact that our operator is not elliptic but transversally elliptic. At a hands-on level, the pole presents a problem in the interpretation of the index - namely, how to compute the Fourier coefficients of this expression. Depending on whether we expand around $q=0$ or $q^{-1}=0$, we will obtain $2 \sum_{n \geq 1} n q^{n}$ or $2 \sum_{n \geq 1} n q^{-n}$, which clearly have different Fourier coefficients. This problem is resolved by giving a certain regularization defined by the behavior of the operator in the neighborhood of each fixed point [17]. Accordingly, we write:

$$
\operatorname{ind}_{\mathrm{vec}}\left(D_{10}\right)=\left[\frac{2 q}{(1-q)^{2}}\right]_{\mathrm{NP}}+\left[\frac{2 q}{(1-q)^{2}}\right]_{\mathrm{SP}} .
$$

Here we have indicated the North Pole and South Pole contributions. As we shall see, the effect of the different regulators in our final results for the determinant will only be in an additive constant which we ignore in the functional determinant.

Hyper multiplets. We do a similar analysis for the hyper multiplets. The fields are separated into $X=\left\{A_{i}^{\alpha}\right\}$ and $\Psi=\left\{\Xi_{i}^{\alpha}\right\}$, with

$$
\Xi_{i}^{\alpha}:=2 \epsilon_{i j}\left(\breve{\breve{\xi}}_{+}^{j} \zeta_{+}^{\alpha}+\overline{\breve{\xi}}_{-}^{j} \zeta_{-}^{\alpha}\right)
$$

again inspired by $[17,33]$. Details of this field splitting can also be found in appendix C. The scalars $A_{i}^{\alpha}$ do not transform under rotations. To compute the charges of the fermions, we note that now it is the spinor $\breve{\xi}_{-}$that vanishes at the North Pole (as can be seen from the expression (C.26)), and therefore the spinor bilinear $\Xi_{i}^{\alpha}$ is in the $2 \times(\mathbf{2}, \mathbf{1})$ of $\mathrm{SO}(4)$, where the factor of 2 counts both $\alpha$ components of a given hypermultiplet. Similarly at the South Pole, $\breve{\xi}_{+}$vanishes and therefore the bilinear is in the $2 \times(\mathbf{1}, \mathbf{2})$ of $\operatorname{SO}(4)$. Putting everything together, we obtain the index for one hyper multiplet:

$$
\operatorname{ind}_{\text {hyp }}\left(D_{10}\right)=\left[-\frac{2 q}{(1-q)^{2}}\right]_{\mathrm{NP}}+\left[-\frac{2 q}{(1-q)^{2}}\right]_{\mathrm{SP}} .
$$

\footnotetext{
${ }^{17}$ Our convention is that a field $\varphi$ of charge $e$ transforms as $\varphi \rightarrow e^{-i e H t} \varphi$.
} 


\section{Zeta function regularization}

We now use the expressions (4.16), (4.18), for the index of the vector and hyper multiplets, to compute their one-loop determinants. Given the infinite product (4.11), we write a formal expression for the logarithm of the one-loop determinant as:

$$
\log \frac{\operatorname{det}_{\Psi} H}{\operatorname{det}_{\mathrm{X}} H}=-\sum_{n \geq 1} a(n) \log \lambda_{n} .
$$

In order to regularize this infinite sum, we use the method of zeta functions. ${ }^{18}$ We first construct the zeta function:

$$
\zeta_{H}(s)=\sum_{n \geq 1} a(n) \lambda_{n}^{-s} .
$$

This converges for suitably large values of $\operatorname{Re}(s)$, and we then analytically continue it to the complex $s$ plane. The superdeterminant (4.19) is then defined as:

$$
\log \frac{\operatorname{det}_{\Psi} H}{\operatorname{det}_{\mathrm{X}} H}=\left.\zeta_{H}^{\prime}(s)\right|_{s=0} .
$$

One of the advantages of the zeta function method is that it easily yields the dependence of the determinant on the physical parameters of the problem. In our case, we have only one parameter in the background which is the overall size of the metric $\ell^{2}=e^{-\mathcal{K}\left(\phi^{I}\right)}$. The dependence on $\ell$ is easily calculated using the scaling properties of the zeta function [51].

We consider the contribution to the index at the north pole and at the south pole separately. At the north pole, we have an expression which is expanded around $q=0$ :

$$
\left[\frac{2 q}{(1-q)^{2}}\right]_{\mathrm{NP}}=2 \sum_{n \geq 1} n q^{n}=\sum_{n \geq 1} 2 n e^{-i t \frac{n}{\ell}} .
$$

In the above language, this index has

$$
a(n)=2 n, \quad \lambda_{n}=\frac{n}{\ell} .
$$

The zeta function for this piece of the determinant is

$$
\zeta_{H}^{\mathrm{NP}}(s)=\sum_{n \geq 1} 2 n\left(\frac{n}{\ell}\right)^{-s}=2 \ell^{s} \zeta_{R}(s-1)
$$

where we have introduced the Riemann zeta function

$$
\zeta_{R}(s)=\sum_{n \geq 1} \frac{1}{n^{s}} .
$$

At the south pole, where we expand in powers of $q^{-1}$, we get a similar expression but the zeta function $\zeta_{H}^{\mathrm{SP}}(s)$ there differs from the north pole answer by a factor of $(-1)^{s}$. We thus

\footnotetext{
${ }^{18}$ The zeta function regularization has been used with great success to compute the perturbative oneloop corrections to the physical quantum gravity path integral (see [51] and follow-ups). Here we use the technique for the exact computation using localization methods.
} 
need to deal with expressions of the type $\log (-n)$, for which we use the positive branch of the logarithm.

Putting together the north pole and the south pole contributions, we obtain

$$
\begin{aligned}
\left.\zeta_{H}^{\prime}(s)\right|_{s=0} & =4 \zeta_{R}^{\prime}(-1)+2 \pi i \zeta_{R}(-1)+4 \zeta_{R}(-1) \log \ell \\
& =4 \zeta_{R}^{\prime}(-1)+2 \pi i \zeta_{R}(-1)+\frac{1}{6} \mathcal{K}\left(\phi^{I}\right) .
\end{aligned}
$$

Since we are not keeping track of purely numerical overall constants, we drop the finite numbers $4 \zeta_{R}^{\prime}(-1)$ and $2 \pi i \zeta_{R}(-1)$ in further expressions. Putting together Equations (4.7), (4.8), (4.21), and (4.26), we obtain:

$$
Z_{1 \text { loop }}^{\text {vec }}\left(\phi^{I}\right)=\exp \left(\mathcal{K}\left(\phi^{I}\right) / 12\right),
$$

with $\mathcal{K}\left(\phi^{I}\right)$ the generalized Kähler potential defined in Equation (2.2).

For the hyper multiplets, we use the same technique, and we find that the index is equal and opposite to that of the vector multiplet - as can be seen directly from the expressions (4.16), (4.18). Our final result is:

$$
Z_{1 \text {-loop }}^{\text {vec }}\left(\phi^{I}\right)=\left(Z_{1 \text {-loop }}^{\text {hyp }}\left(\phi^{I}\right)\right)^{-1}=\exp \left(\mathcal{K}\left(\phi^{I}\right) / 12\right) .
$$

Although we have only worked out the details of the vector and hyper multiplets, it is clear that the above calculation will also go through essentially unchanged once we have fixed the off-shell complex of any multiplet. Since there is only one scale set by $e^{-\mathcal{K}}$ in the localization background, the functional determinant will have the symplectically invariant form $e^{-a_{0} \mathcal{K}\left(\phi^{I}\right)}$. The number $a_{0}$ receives contributions from each multiplet of the $\mathcal{N}=2$ supergravity theory:

$$
a_{0}=a_{0}^{\text {grav }}+n_{3 / 2} a_{0}^{3 / 2}+\left(n_{\mathrm{v}}+1\right) a_{0}^{\mathrm{vec}}+n_{\mathrm{h}} a_{0}^{\text {hyp }},
$$

where $n_{3 / 2},\left(n_{\mathrm{v}}+1\right), n_{\mathrm{h}}$ are the number of gravitini, vectors and hypers in the off-shell theory, respectively. From our results in this section, $a_{0}^{\text {vec }}=-a_{0}^{\text {hyp }}=-1 / 12$. As mentioned in the introduction, we are currently investigating the details of the off-shell computation of the graviton and gravitini determinants [30]. We will see in the following section how we can use existing on-shell computations to check our formula (4.28) for the vectors and hypers, as well as to deduce the coefficients $a_{0}$ for the other multiplets.

\section{Relation to previous results for the black hole entropy}

The leading logarithmic corrections to the classical black hole entropy have been obtained in $[47,52,53]$ by explicitly evaluating the one-loop determinant of the kinetic terms of all the quadratic fluctuations of the theory around the classical attractor background (2.4). This is a very intricate computation that needs a diagonalization of the kinetic terms of all the fields of the theory, and it depends on the fact that the values of the metric, fluxes and scalars in the attractor solution are related by supersymmetry. ${ }^{19}$ In contrast, the

\footnotetext{
${ }^{19}$ The recent interesting work of [54,55] also uses on-shell techniques, but relies only on the chiral spectrum of the supersymmetry algebra.
} 
localization method involves the one-loop determinant of the deformation operator $Q \mathcal{V}$, which does not depend on the equations of motion and the associated kinetic mixings. At a practical level, the on-shell computation of $[47,52,53]$ proceeds by solving for the spectrum of eigenvalues of the various Laplacians of the theory, and observing that there is a huge cancellation among them. The index theorem, on the other hand, reduces this problem to a very simple computation at the fixed points of a certain U(1) action.

The results of the on-shell and off-shell methods agree in the large-charge limit, as expected. In fact a little more can be said about the interplay of the techniques used in these two approaches. In this section we present our understanding of this relationship. Using these relations, we also explain the cancellations regarding $\frac{1}{8}$-BPS black holes in $\mathcal{N}=8$ string theories that were observed in [13].

\section{Relation to large-charge on-shell computations}

We consider a limit in which all the charges $\left(q_{I}, p^{I}\right)$ scale uniformly by a large parameter $\Lambda$, i.e. $\left(q_{I}, p^{I}\right) \rightarrow \Lambda\left(q_{I}, p^{I}\right)$. In the leading $\Lambda \rightarrow \infty$ limit, one can evaluate the quantum entropy integral (1.4) using the saddle point method. If we ignore the determinant factor $Z_{\text {det }}$, the saddle point equations are simply the extremization equations of the exponent of (1.4). As we discussed in section 2, these extremization equations are precisely the attractor equations (2.7), and the saddle point values $\phi_{*}^{I}=\operatorname{Re} X_{*}^{I}$, the attractor value of the scalar fields.

From the attractor equations (2.7), we see that the attractor values $\phi_{*}^{I} \sim \Lambda$ for large $\Lambda$, and the attractor entropy (2.10) scales as $\Lambda^{2}$. From Equation (2.2), we see that the determinants (4.28) scale as $\Lambda^{-2 a_{0}}$ and therefore they will contribute to the entropy as $\log \Lambda$, so that it is indeed justified to ignore them to leading order. The resulting classical entropy is:

$$
S_{\mathrm{BH}}^{\text {class }}=\pi\left(-q_{I} e_{*}^{I}-4 \operatorname{Im} F^{(0)}\left(\left(e_{*}^{I}+i p^{I}\right) / 2\right)\right) \approx \frac{A_{H}}{4},
$$

where $F^{(0)}$ denotes the prepotential without any $\widehat{A}$-dependence, corresponding to the twoderivative effective action, which is consistent with the large-charge approximation. This entropy agrees with the attractor mechanism (1.1), (1.2).

The first corrections to the leading large-charge entropy are given by the first corrections to the saddle point value (5.1), of (1.4). In the large-charge limit, we know that $A_{H} \sim e^{-\mathcal{K}} \sim \Lambda^{2}$. From Equation (4.28) we deduce that

$$
S_{\mathrm{BH}}^{\mathrm{qu}}=\frac{A_{H}}{4}+a_{0} \log A_{H}+\cdots,
$$

where the number $a_{0}$ is precisely the coefficient defined in (4.29). In section 4 , we saw that

$$
a_{0}^{\mathrm{vec}}=-a_{0}^{\mathrm{hyp}}=-\frac{1}{12},
$$

which indeed agrees with the corresponding on-shell computations of the log corrections to the black hole entropy [47], performed using the heat-kernel method. In the rest of this section, we make some comments on the relation between our exact index calculation of section 4 , heat-kernels, and the large-charge expansion. 
The heat-kernel method (see e.g. [56]) to compute the functional determinant of an operator $D$ uses its representation as an integral over the proper time $t$ :

$$
\frac{1}{2} \log \operatorname{det}(D)=-\frac{1}{2} \int_{0}^{\infty} \frac{d t}{t} K(t, D), \quad K(t, D)=\operatorname{Tr} e^{-D t} .
$$

The integral on the right-hand side of (5.4) is not always well-defined. The divergences as $t \rightarrow 0$ arises from the UV divergences, for which we assume a UV cutoff $\varepsilon$. The divergences as $t \rightarrow \infty$ appear because of zero or negative eigenvalues of $D$. In our problem, the relevant operator $H$ does not have any negative eigenvalues, nor does it have zero modes since, due to the boundary conditions we impose, the ghost-for-ghost fields are absent.

The coefficient of the logarithmic term in a large-charge expansion of quantum black hole entropy (1.3) is determined by the constant coefficient in the $t \rightarrow 0$ expansion of the integrated heat-kernel [47]. Our calculations of section 4 can be written as (here $q=e^{-t}$ ):

$$
\frac{1}{2} \log \operatorname{det}_{\mathrm{vec}}(H)=-\frac{1}{2} \log \operatorname{det}_{\mathrm{hyp}}(H)=\int_{\varepsilon}^{\infty} \frac{d t}{t} \frac{2 q}{(1-q)^{2}} .
$$

If we are only interested in the logarithmic term in the large charge expansion, we can also directly using the $t \rightarrow 0$ expansion of the heat kernel in the above integrals:

$$
\frac{2 q}{(1-q)^{2}}=\frac{2}{t^{2}}-\frac{1}{6}+\frac{t^{2}}{120}+O(t)^{4}
$$

from which we recover the result (5.3) for the coefficient $a_{0}$.

We defined the number $a_{0}$ as appearing in the off-shell one-loop determinant in section 4, and we saw above that the same number is the coefficient of the logarithmic correction to the large-charge expansion of black hole entropy. We can actually use this consistency between on-shell and off-shell methods to deduce the value of $a_{0}$ for the graviton and gravitini multiplets. The results of [47] demand that $a_{0}^{3 / 2}=-\frac{11}{12}$, and $a_{0}^{\text {grav }}=2$ in the gauge $\sqrt{-g}=1$.

\section{Miraculous cancellation in truncations of extended supergravities}

In [13], the $\frac{1}{8}$-BPS black hole in $\mathcal{N}=8$ theory was considered from both the macroscopic and microscopic point of view. The physical low energy macroscopic field content is that of an $\mathcal{N}=8$ graviton multiplet which, in the $\mathcal{N}=2$ language that we are considering, consists of one $\mathcal{N}=2$ graviton multiplet, $n_{3 / 2}=6$ gravitini multiplets, $n_{\mathrm{v}}=15$ vector multiplets, and $n_{\mathrm{h}}=10$ hyper multiplets. The macroscopic entropy was computed using localization in [13] in the truncated theory that was first considered in [57], where the physical spectrum consists only of the $\mathcal{N}=2$ graviton multiplet coupled to $n_{\mathrm{v}}^{\text {trun }}=7$ vector multiplets.

In this truncated theory, only the measure for the zero-modes of $Q$ was taken into account in [13], and it was computed to be $Z_{0}=e^{\left(n_{\mathrm{v}}^{\text {trun }}+1\right) \mathcal{K} / 2} \times O\left(\Lambda^{0}\right)$. Assuming further that the non-zero mode determinant $Z_{\text {det }}^{\prime}=1$ the formula derived was:

$$
W^{\text {pert }}(\Delta)=\sqrt{2} \pi \frac{1}{\Delta^{7 / 4}} I_{7 / 2}(\pi \sqrt{\Delta})
$$


where $\Delta$ is the unique quartic U-duality invariant of the charges of the $\mathcal{N}=8$ theory. This formula was seen to agree on the nose with the microscopic formula for the black hole degeneracy [13].

We now have a better understanding of this agreement. Let us split the contribution of one vector multiplet into two parts as $a_{0}^{\mathrm{vec}}=-\frac{1}{12}=-\frac{1}{2}+\frac{5}{12}$, where the $-\frac{1}{2}$ is the contribution considered in [13], and $\frac{5}{12}$ is the rest. Then, using the values of $a_{0}$ for the various multiplets written in the previous subsection, the contribution to $a_{0}$ ignored in [13] is

$$
\frac{5}{12}\left(n_{\mathrm{v}}^{\text {trun }}+1\right)-\frac{1}{12}\left(n_{\mathrm{v}}-n_{\mathrm{v}}^{\text {trun }}\right)+\frac{1}{12} n_{\mathrm{h}}-\frac{11}{12} n_{3 / 2}+2 .
$$

For the field content of the $\mathcal{N}=8$ theory and the $\mathcal{N}=2$ truncation as given above, this indeed adds up to zero, thus explaining the miraculous cancellation in the full string theory seen in [13]. This cancellation can already be seen at the leading log level from the results of [47]. It is now clear from the comments in this section that this cancellation holds to all orders in perturbation theory.

We can also consider $\mathcal{N}=4$ string theories, where the physical low energy macroscopic field content is an $\mathcal{N}=4$ graviton multiplet coupled to $N_{\mathrm{v}} \mathcal{N}=4$ vector multiplets. In terms of $\mathcal{N}=2$ multiplets, we have one graviton multiplet, $n_{3 / 2}=2$ gravitini multiplets, $n_{\mathrm{v}}=N_{\mathrm{v}}+1$ vector multiplets, and $n_{\mathrm{h}}=N_{\mathrm{v}}$ hyper multiplets. The total logarithmic correction according to (4.29) is given by $a_{0}=2-\frac{11}{12} \times 2-\frac{1}{12} \times 2=0$, as consistent with the on-shell computations in the limit when all the charges are scaled to be equally large. We can also consider a truncation in which we have an agreement for the leading Bessel function in the Cardy limit [58].

\section{Exact formulas for $\mathcal{N}=2$ quantum black hole entropy and the relation to topological strings}

The true power of the localization method clearly lies in the fact that one can go beyond the perturbative large charge approximation to get an exact result for black hole entropy. In this section we propose such an exact entropy formula for BPS black holes in $\mathcal{N}=2$ supergravity coupled to $n_{\mathrm{v}}$ vector multiplets and $n_{\mathrm{h}}$ hyper multiplets. We then make some comments relating our formula to the microscopic formula of [28], as well as on some relations with topological string theory.

In the previous sections, we have seen that one-loop determinant of the fluctuations around the localization manifold takes the symplectically invariant form: ${ }^{20}$

$$
Z_{1 \text {-loop }}=\exp \left(-\mathcal{K}\left(\phi^{I}\right)\left(2-\frac{\chi}{24}\right)\right), \quad \chi=2\left(n_{\mathrm{v}}+1-n_{\mathrm{h}}\right) .
$$

Recall from the discussion below Equation (1.4) that the full determinant factor in the exact formula has two contributing pieces - the one-loop fluctuations $Z_{1 \text {-loop }}$, and the measure

\footnotetext{
${ }^{20}$ In this section we assume $a_{0}^{\text {grav }}=2$ (as argued for above) in the gauge $\sqrt{-g}=1$ which we use throughout this paper. It is important to derive this result from a proper analysis of the fluctuating Weyl multiplet and the corresponding gauge-fixing. This is under investigation [30].
} 
from the curvature of field space itself $Z_{\mathrm{det}}^{\text {ind }}$. Combining these elements, we obtain:

$$
W^{\text {pert }}(q, p)=\int_{\mathcal{M}_{Q}} \prod_{I=0}^{n_{\mathrm{v}}} d \phi^{I} e^{-\pi q_{I} \phi^{I}+4 \pi \operatorname{Im} F\left(\left(\phi^{I}+i p^{I}\right) / 2\right)} e^{-\mathcal{K}\left(\phi^{I}\right)(2-\chi / 24)} Z_{\mathrm{det}}^{\text {ind }} .
$$

To move on, we need to discuss the details of the prepotential function $F\left(X^{I}, \widehat{A}\right)$, which is a holomorphic homogeneous function of weight 2 in its variables under the scalings $X^{I} \rightarrow$ $\lambda X^{I}, \widehat{A} \rightarrow \lambda^{2} X^{I}$. Generically, we have an expansion of the form:

$$
F\left(X^{I}, \widehat{A}\right)=\sum_{g=0}^{\infty} F^{(g)}\left(X^{I}\right) \widehat{A}^{g}
$$

that enters the Wilsonian effective action of the on-shell supergravity. The function $F^{(0)}\left(X^{I}\right)$ controls the two-derivative interactions, and the coefficients $F^{(g)}, g \geq 1$, describe higher derivative couplings of the form $C^{2} T^{2 g-2}$ and terms related by supersymmetry, where $C$ is related to the Weyl tensor, and $T$ is related to the graviphoton field strength.

At the two-derivative level, the prepotential has the form

$$
F^{(0)}\left(X^{I}\right)=-\frac{1}{2} \sum_{i, j, k=1}^{n_{\mathrm{v}}} C_{i j k} \frac{X^{i} X^{j} X^{k}}{X^{0}}
$$

for a choice of symmetric $C_{i j k}$. At this level, we can think of the measure of the scalars in a geometric manner, and compute it from the knowledge of the two-derivative kinetic term of the scalar sigma model. To be more thorough, we should take into account all the fields in the theory - this can be done by using duality invariance as a criterion for the measure as in [59]. Both these approaches give rise to the measure:

$$
Z_{\operatorname{det}}^{\text {ind }}=\left(\operatorname{det} \operatorname{Im}\left(F_{I J}^{(0)}\right)\right)^{\frac{1}{2}} .
$$

For a prepotential of the form (6.4), and for $^{21} p^{0}=0, q_{0} \neq 0$, we can compute the various expressions entering the exact formula (6.2). We have:

$$
e^{-\mathcal{K}^{(0)}}=\frac{C_{i j k} p^{i} p^{j} p^{k}}{\phi^{0}}
$$

and $\operatorname{det} \operatorname{Im}\left(F_{I J}^{(0)}\right)=A /\left(\phi^{0}\right)^{\left(n_{\mathrm{v}}+3\right) / 2}$ where $A$ does not depend on $\phi^{I}$ (but does depend on $C_{i j k}$ and $p^{i}$ ). However, using these expressions in our integral expression (6.2) leads to a formula which does not match the corresponding microscopic BPS state counting formulas beyond the leading logarithmic correction (see e.g. [28, 34, 35]).

From our point of view, this discrepancy arises from our lack of complete understanding of the induced measure term. The current best understanding of the measure in the supergravity field space comes from the work of [29, 59], whose main guiding principle

\footnotetext{
${ }^{21}$ In the type IIA setting, this means absence of D6-branes in the charge configuration making up the black hole.
} 
is duality invariance. These authors have argued that imposing duality invariance leads to a non-holomorphic modification to the induced measure. At the two-derivative level, including these corrections, one has:

$$
Z_{\text {det }}^{\text {ind }}=\left(\phi_{0}^{-2} \exp \left(-\mathcal{K}^{(0)}\left(\phi^{I}\right)\right)\right)^{\frac{\chi}{24}-1},
$$

We note that the precise context in which these modifications have been derived is different from the one considered here. Notwithstanding this difference, if we combine the expression (6.7) and the one-loop factor (6.1) in our exact formula (6.2), we obtain:

$$
W^{\text {pert }}(q, p)=\int_{\mathcal{M}_{Q}} \prod_{I=0}^{n_{\mathrm{v}}} d \phi^{I} \exp \left(-\pi q_{I} \phi^{I}+4 \pi \operatorname{Im} F^{(0)}\left(\left(\phi^{I}+i p^{I}\right) / 2\right)\right)\left(\phi^{0}\right)^{2-\frac{\chi}{12}} e^{-\mathcal{K}^{(0)}(\phi)} .
$$

The black hole entropy formula conjectured in the paper [28] based on consistency with the Rademacher expansion of the microscopic black hole degeneracies in string theory has exactly the same form as (6.8), with the two-derivative expressions $F^{(0)}, \mathcal{K}^{(0)}$ replaced by the all-order expressions $F, \mathcal{K}$, respectively.

To go beyond the two-derivative level in our formalism, we need a formula for the induced measure at all orders. The work of $[29,59]$ provides a formalism to take into account all the holomorphic corrections to the supergravity measure. More work, however, needs to be done to fully understand the non-holomorphic effects in the induced measure as defined in our treatment. It is possible that the a priori induced measure in the original supergravity path integral suffers from a holomorphic anomaly. Similar ideas have been proposed in [60] in the context of the topological string theory. A computation of this a priori measure from first principles would complete the derivation of the exact quantum black hole entropy in the gravitational theory.

\section{Comments on relations to topological string theory}

Consider type IIA string theory compactified on a Calabi-Yau 3-fold $C Y_{3}$. The A-model topological string partition function on $C Y_{3}$ has the expression:

$$
F_{\text {top }}=-i \frac{(2 \pi)^{3}}{6 \lambda^{2}} C_{i j k} t^{i} t^{j} t^{k}-\frac{i \pi}{12} c_{2 i} t^{i}+F_{G W}\left(\lambda, t^{i}\right),
$$

where $\lambda$ is the topological string coupling, $t^{i}$ are the moduli fields (the complexified Kähler structure in the type IIA theory), $c_{2 i}$ are the second Chern classes of the 4-cycles of the $C Y_{3}$, and $F_{G W}$ is the generating function of the Gromov-Witten $(\mathrm{GW})$ invariants of the $C Y_{3}$ that admits an expansion in powers of $\lambda$. By comparing (6.9) to the corresponding Wilsonian expression (6.3) in the supergravity, we obtain: ${ }^{22}$

$$
F_{\text {top }}=\frac{i \pi}{2} F, \quad t^{i}=\frac{X^{i}}{X^{0}}, \quad \lambda^{2}=\frac{\pi^{2}}{8} \frac{\widehat{A}}{\left(X^{0}\right)^{2}} .
$$

\footnotetext{
${ }^{22}$ There are important subtleties associated with the above identification, having to do with the action of duality (symplectic transformations) on the geometry of the Calabi-Yau surface and in supergravity [29, 61]. We do not add anything to this discussion.
} 
The value of the topological string coupling constant on the supergravity localization manifold analyzed in this paper is $|\lambda|=2 \pi \sqrt{2} / \phi^{0}$ - which is small for large values of the charges. The microscopic analysis of $[28,34,35]$ is based on large $\lambda$. Using the relation of the GW invariants to the Gopakumar-Vafa invariants related to counting M2-branes in M-theory, then making a precise prediction for the degenerate instanton contribution at large topological string coupling, and a subsequent analytic continuation, the authors of $[28,34,35]$ claimed that the the topological string partition function at weak coupling must have an additional logarithmic term:

$$
\widetilde{F}_{\text {top }}=-i \frac{(2 \pi)^{3}}{6 \lambda^{2}} C_{A B C} t^{A} t^{B} t^{C}-\frac{\chi}{24} \log \lambda-\frac{i \pi}{12} c_{2 A} t^{A}+F_{G W}\left(\lambda, t^{A}\right) .
$$

where $\chi$ is the Euler characteristic of the Calabi-Yau three-fold. The puzzle then is to interpret the logarithmic term in supergravity. Being a non-local contribution, it cannot arise at any order in perturbation theory in $\widehat{A}$.

From our point of view, the logarithmic contribution in $\lambda$ (or equivalently in $\widehat{A}$ ) appears as a quantum effect. If we interpret the formula (6.8) as an OSV type formula, then the imaginary part of the prepotential contains precisely the additional non-local logarithmic piece with coefficient $\chi / 24$ that is predicted by the analytic continuation of the microscopic theory. (We recall that in a string compactification on a $C Y_{3}$, the number $\chi=2\left(n_{\mathrm{v}}+1-n_{\mathrm{h}}\right.$ ) is the Euler characteristic of the $C Y_{3}$.) Our $A d S_{2}$ functional integral incorporates the integration over massless modes, and although the Wilsonian action of supergravity does not contain the logarithmic term, the effective 1PI action appearing in the exponent of Equation (6.8) does. ${ }^{23,24}$ We mention that most of this interpretation can be reconstructed by combining the duality arguments of $[29,61]$ with the computation of the leading logarithmic effects of [47]. The one point we add to this discussion is the direct calculation of the one-loop effects proportional to $e^{-a_{0} \mathcal{K}}$.

Finally, we note that, in addition to being at different values of coupling constants, the values of the moduli in our analysis and that of [28] are also different. The authors of [28] work with moduli $t_{\infty}$ in asymptotically flat space, while we choose attractor values of moduli to define the black hole degeneracy since we are only interested in the singlecenter black holes. Our results could be interpreted to mean that the relevant index does not suffer any wall-crossing on moving from one regime to the other.

These results may also point to a new "black hole index" that is simply constant over all of moduli space. Indeed, an argument was made in $[65,66]$ that, when a black hole preserves at least four supercharges and consequently at least an $\mathrm{SU}(2)_{R}$ symmetry at its horizon, its quantum entropy is equal to a supersymmetric index. Defining this index in the microscopic theory is not an easy problem, but one can do that in $\mathcal{N}=4$ string theories. In that case the black hole index is given by the coefficient of a mock modular form, defined using the attractor value of moduli, and it is constant all over of moduli

\footnotetext{
${ }^{23} \mathrm{~A}$ deeper explanation of this phenomenon appears in [62].

${ }^{24}$ There are similar $\log g_{s}$ terms in the couplings of the low energy effective action of string theory in flat space, e.g. [63], which can be explained by mixing between the local and non-local part of the 1PI action when rescaling from string frame to Einstein frame [64].
} 
space [67]. A similar phenomenon in $\mathcal{N}=2$ string theories would point towards a larger symmetry underlying the BPS states of $\mathcal{N}=2$ theories as proposed in [68, 69].

\section{Acknowledgments}

We would like to thank Daniel Butter, Stefano Cremonesi, Bernard de Wit, Atish Dabholkar, João Gomes, Mahir Hazdic, Gianluca Inverso, Sungjay Lee, Hirosi Ooguri, Boris Pioline, Ashoke Sen, and especially Rajesh Gupta and Imtak Jeon for useful and informative conversations. This work is supported by the ERC Advanced Grant no. 246974, "Supersymmetry: a window to non-perturbative physics" and the EPSRC First Grant UK EP/M018903/1.

\section{A Conventions}

The summation convention for $\mathrm{SU}(2)$ indices is NW-SE and (anti)symmetrization of indices is done with weight one. The antisymmetric tensor of $\mathrm{SU}(2)$ is such that

$$
\epsilon^{i j} \epsilon_{j k}=-\delta_{k}^{i} \text { and } \epsilon^{i j} \epsilon_{i j}=2 .
$$

We take the following hermitian tangent space Dirac matrices in Euclidean signature:

$$
\gamma_{1}=\sigma_{1} \otimes 1, \quad \gamma_{2}=\sigma_{2} \otimes 1, \quad \gamma_{3}=\sigma_{3} \otimes \sigma_{1}, \quad \gamma_{4}=\sigma_{3} \otimes \sigma_{2},
$$

where $\sigma_{i}, i=1,2,3$ are the Pauli matrices. We also define the usual combination $\gamma^{a b}=$ $\frac{1}{2}\left[\gamma^{a}, \gamma^{b}\right]$ and similarly for higher-rank $\gamma$ matrices. In addition, $\gamma_{5}=-\gamma_{1} \gamma_{2} \gamma_{3} \gamma_{4}$. These matrices obey the following useful identities in four dimensions:

$$
\begin{array}{rlrl}
\gamma_{a b c} & =\epsilon_{a b c d} \gamma^{d} \gamma_{5}, & \gamma_{a b c d} & =-\epsilon_{a b c d} \gamma_{5}, \\
\gamma^{a b} \gamma_{b} & =3 \gamma^{a}, & \gamma^{a b c} \gamma_{c} & =2 \gamma^{a b}, \\
\gamma_{b} \gamma^{a} \gamma^{b} & =-2 \gamma^{a}, & \gamma_{c} \gamma^{a b} \gamma^{c} & =0, \\
\gamma_{a} \gamma_{b c} & =\delta_{a b} \gamma_{c}-\delta_{a c} \gamma_{b}+\epsilon_{a b c d} \gamma^{d} \gamma_{5} . &
\end{array}
$$

We define the Majorana conjugate of a spinor $\lambda$ as

$$
\bar{\lambda} \equiv \lambda^{T} \mathcal{C}
$$

where the charge conjugation matrix is given by $\mathcal{C} \equiv \sigma_{2} \otimes \sigma_{1}$ in the Clifford algebra representation (A.2). For a Majorana spinor carrying an extra SU(2) index (e.g. because of R-symmetry), the symplectic Majorana reality condition reads

$$
\left(\lambda^{i}\right)^{*}=\epsilon_{i j} \mathcal{C}^{T} \lambda^{j}
$$

This condition can be imposed consistently in 4-dimensional Euclidean space, along with a chirality (Weyl) projection.

For two spinors $\chi$ and $\lambda$, we have the so-called "Majorana flip relation":

$$
\bar{\chi} \gamma_{(r)} \lambda= \pm t_{r} \bar{\lambda} \gamma_{(r)} \chi,
$$


where $\gamma_{(r)}$ is a Dirac matrix of rank $r$, and the plus sign holds when both $\chi$ and $\lambda$ are anti-commuting (Grassmann-odd). In 4-dimensional Euclidean space, we take

$$
t_{0}=1, \quad t_{1}=-1, \quad t_{2}=-1, \quad t_{3}=1 \quad \text { and } t_{r+4}=t_{r} .
$$

We denote the (anti)self-dual part of an $\mathrm{SU}(2)$ antisymmetric tensor as

$$
T_{a b}^{-}=T_{a b}^{i j} \epsilon_{i j}, \quad T_{a b}^{+}=T_{a b i j} \epsilon^{i j} .
$$

A useful property of spinors and antisymmetric tensors is that when $T_{a b} \gamma^{a b}$ acts on a spinor of (positive) negative chirality, it is projected onto its (anti)self-dual part:

$$
T_{a b} \gamma^{a b} \xi_{+}^{i}=T_{a b}^{-} \gamma^{a b} \xi_{+}^{i} \quad \text { and } T_{a b} \gamma^{a b} \xi_{-}^{i}=T_{a b}^{+} \gamma^{a b} \xi_{-}^{i}
$$

\section{Analytic continuation to Euclidean space}

The $\mathcal{N}=2$ superconformal algebra that we use [23, 24] holds for theories in Minkowski signature. We need to adapt it to our problem of computing a Euclidean path integral, for which we follow the approach of [17]. The idea is to use the original Minkowski algebra to perform algebraic computations such as computing the field variations, the action, and the symbol in appendix $\mathrm{C}$, and then perform an analytic continuation to Euclidean space, which we describe below, at the very end of the algebraic computations. In this paper, we have indicated this procedure by the inner product $(.,$.$) , and some explicit formulas are given$ in the symbol computation. This procedure could be streamlined by directly developing a Euclidean algebra from the beginning, this problem is currently being addressed.

The Euclidean continuation is performed via the usual $t \rightarrow i \tau$ in the metric and field configuration, and we regard Minkowski complex conjugates as independent fields in Euclidean space, i.e. $X^{I}$ and $\bar{X}^{I}$ and are two independent scalars, $\Omega_{i}$ is independent of $\Omega^{i}$, and so on. Moreover, in 4-dimensional spacetime with Minkowski signature, fundamental spinors are Weyl or Majorana spinors, whereas in Euclidean signature they are symplectic Majorana-Weyl [40]. To accommodate for this change of reality property when continuing $\mathcal{N}=2$ superconformal gravity to Euclidean signature, we follow the method of [12] and introduce new spinors $\xi_{ \pm}^{i}$ to parametrize the supersymmetry transformations, where $i=1,2$ is an $\mathrm{SU}(2)$ index and \pm denotes the chirality of the spinor. S-supersymmetry is also parametrized with Euclidean spinors $\eta_{ \pm}^{i}$. We replace the Minkowski spinor parameters entering the algebra according to:

$$
\epsilon_{i} \rightarrow \epsilon_{i j} \xi_{-}^{j} ; \epsilon^{i} \rightarrow \xi_{+}^{i}, \quad \text { and } \quad \eta_{i} \rightarrow \epsilon_{i j} \eta_{+}^{j} ; \eta^{i} \rightarrow \eta_{-}^{i} .
$$

By definition, these spinors obey the symplectic Majorana condition

$$
\left(\xi_{ \pm}^{i}\right)^{*}=-\epsilon_{i j}\left(\sigma_{2} \otimes \sigma_{1}\right) \xi_{ \pm}^{j}
$$

and similarly for $\eta_{ \pm}^{i}$. In this paper, we will take the spinors parametrizing the supersymmetry transformations to be Grassmann-even (commuting) spinors. This can be achieved by extracting a Grassmann number on both sides of the transformation rules. We may then consider successive supersymmetry transformations with equal parameters directly. This is useful since, as explained in the introduction of this paper, we will be interested in writing the action of the supercharge $Q$ generated by a specific parameter, chosen so that $Q$ squares to $\left(L_{0}-J_{0}\right)$ which is the relevant supersymmetry for localization. 
Vector multiplets. In a similar fashion, we introduce new Euclidean spinors $\lambda_{ \pm}^{i}$ to analytically continue the spinors of the vector multiplets (here we do not extract a Grassmann number from the spinors so the $\lambda_{ \pm}^{i}$ are still anti-commuting)

$$
\Omega_{i}^{I} \rightarrow \epsilon_{i j} \lambda_{+}^{I j} \quad \text { and } \quad \Omega^{I i} \rightarrow-\lambda_{-}^{I i} .
$$

Hyper multiplets. We introduce the following spinors $\zeta_{ \pm}^{\alpha}$ to continue the hyperini (which are also still anti-commuting)

$$
\zeta^{\alpha} \rightarrow \zeta_{-}^{\alpha} \quad \text { and } \quad \zeta_{\alpha} \rightarrow \rho_{\alpha \beta} \zeta_{+}^{\beta},
$$

where $\rho_{\alpha \beta}$ is a skew-symmetric constant matrix [42] satisfying

$$
\rho_{\alpha \beta} \rho^{\beta \gamma}=-\delta_{\alpha}^{\gamma} \quad \text { with } \quad \rho^{\alpha \beta} \equiv\left(\rho_{\alpha \beta}\right)^{*} .
$$

\section{B Killing spinors of $A d S_{2} \times S^{2}$}

In this appendix, we review the solutions to the BPS equations of $\mathcal{N}=2$ supergravity:

$$
\begin{gathered}
2 D_{\mu} \xi_{ \pm}^{i} \pm \frac{1}{16} T_{a b}^{\mp} \gamma^{a b} \gamma_{\mu} \xi_{\mp}^{i}-\gamma_{\mu} \eta_{\mp}^{i}=0 \\
\gamma^{\mu} D_{\mu} T_{a b}^{\mp} \gamma^{a b} \xi_{\mp}^{i} \pm 24 D \xi_{ \pm}^{i}-T_{a b}^{\mp} \gamma^{a b} \eta_{ \pm}^{i}=0 .
\end{gathered}
$$

The solutions to these equations with $A d S_{2} \times S^{2}$ boundary conditions, the localizing manifold of our problem, were found and analyzed in $[12,14]$. We rewrite the solutions in our conventions so as to have an easy reference for some calculations in sections 2 and 4 .

As explained in section 2, the metric and $T$-tensor appearing in (B.1), (B.2) are given by (2.4), (2.5) in the $\sqrt{-g}=1$ gauge. We now observe that a set of solutions to the conformal Killing spinor equations can be found simply by setting

$$
\eta_{ \pm}^{i}=0
$$

taking $\xi_{ \pm}^{i}$ to be a solution of

$$
\begin{aligned}
& 2 D_{\mu} \xi_{+}^{i}+\frac{1}{16} T_{a b}^{-} \gamma^{a b} \gamma_{\mu} \xi_{-}^{i}=0 \\
& 2 D_{\mu} \xi_{-}^{i}-\frac{1}{16} T_{a b}^{+} \gamma^{a b} \gamma_{\mu} \xi_{+}^{i}=0
\end{aligned}
$$

with $A d S_{2} \times S^{2}$ boundary conditions, and with the field $D$ satisfying (with $\xi^{i}=\xi_{+}^{i}+\xi_{-}^{i}$ )

$$
\left(24 D \gamma_{5}+\gamma^{\mu} D_{\mu} T_{a b} \gamma^{a b}\right) \xi^{i}=0
$$

Note that in (B.4), the covariant derivative only contains the spin-connection in our gaugefixed theory since $b_{\mu}$ and the R-symmetry gauge fields have been set to zero, i.e., $D_{\mu} \xi_{ \pm}^{i}=$ $\partial_{\mu} \xi_{ \pm}^{i}-\frac{1}{4} \omega_{\mu}^{a b} \gamma_{a b} \xi_{ \pm}^{i}$. 
For our black hole solution in the gauge $\sqrt{-g}=1$, we have

$$
T_{a b}^{-}=\left(\begin{array}{cccc}
0 & 4 i & 0 & 0 \\
-4 i & 0 & 0 & 0 \\
0 & 0 & 0 & 4 i \\
0 & 0 & -4 i & 0
\end{array}\right), \quad T_{a b}^{+}=\left(\begin{array}{cccc}
0 & 4 i & 0 & 0 \\
-4 i & 0 & 0 & 0 \\
0 & 0 & 0 & -4 i \\
0 & 0 & 4 i & 0
\end{array}\right)
$$

and (B.4) reduces to

$$
D_{\mu} \xi^{i}=-\frac{1}{2}\left(\sigma_{3} \otimes 1\right) \gamma_{\mu} \gamma_{5} \xi^{i}
$$

The solutions to (B.7) have been obtained for general $A d S_{n} \times S^{m}$ geometries in [70]. We parametrize the Euclidean $A d S_{2} \times S^{2}$ space as

$$
d s^{2}=g_{\mu \nu} d x^{\mu} d x^{\nu}=\sinh ^{2} \eta d \tau^{2}+d \eta^{2}+d \psi^{2}+\sin ^{2} \psi d \phi^{2}
$$

to obtain the following four complex, linearly independent solutions of (B.7):

$$
\begin{gathered}
\xi_{1}=\sqrt{2} e^{\frac{i}{2}(\phi+\tau)}\left(\begin{array}{c}
\sinh \frac{\eta}{2} \cos \frac{\psi}{2} \\
i \cosh \frac{\eta}{2} \cos \frac{\psi}{2} \\
-\sinh \frac{\eta}{2} \sin \frac{\psi}{2} \\
i \cosh \frac{\eta}{2} \sin \frac{\psi}{2}
\end{array}\right), \quad \xi_{2}=\sqrt{2} e^{-\frac{i}{2}(\phi+\tau)}\left(\begin{array}{c}
i \cosh \frac{\eta}{2} \sin \frac{\psi}{2} \\
-\sinh \frac{\eta}{2} \sin \frac{\psi}{2} \\
i \cosh \frac{\eta}{2} \cos \frac{\psi}{2} \\
\sinh \frac{\eta}{2} \cos \frac{\psi}{2}
\end{array}\right) \\
\xi_{3}=\sqrt{2} e^{\frac{i}{2}(\phi-\tau)}\left(\begin{array}{c}
\cosh \frac{\eta}{2} \cos \frac{\psi}{2} \\
i \sinh \frac{\eta}{2} \cos \frac{\psi}{2} \\
-\cosh \frac{\eta}{2} \sin \frac{\psi}{2} \\
i \sinh \frac{\eta}{2} \sin \frac{\psi}{2}
\end{array}\right), \quad \xi_{4}=\sqrt{2} e^{-\frac{i}{2}(\phi-\tau)}\left(\begin{array}{c}
i \sinh \frac{\eta}{2} \sin \frac{\psi}{2} \\
-\cosh \frac{\eta}{2} \sin \frac{\psi}{2} \\
i \sinh \frac{\eta}{2} \cos \frac{\psi}{2} \\
\cosh \frac{\eta}{2} \cos \frac{\psi}{2}
\end{array}\right) .
\end{gathered}
$$

Spinors in Euclidean signature have a symplectic Majorana reality condition, and from the above complex solutions to (B.7), one may generate symplectic Majorana solutions by taking the following combinations

$$
\begin{array}{llll}
\xi_{(1)}^{i}=\left(\xi_{1},-i \xi_{2}\right), & \xi_{(2)}^{i}=\left(i \xi_{1},-\xi_{2}\right), & \xi_{(3)}^{i}=\left(\xi_{2}, i \xi_{1}\right), & \xi_{(4)}^{i}=\left(i \xi_{2}, \xi_{1}\right), \\
\xi_{(5)}^{i}=\left(\xi_{3},-i \xi_{4}\right), & \xi_{(6)}^{i}=\left(i \xi_{3},-\xi_{4}\right), & \xi_{(7)}^{i}=\left(\xi_{4}, i \xi_{3}\right), & \xi_{(8)}^{i}=\left(i \xi_{4}, \xi_{3}\right),
\end{array}
$$

where the $\mathrm{SU}(2)$ notation is $\xi^{i}=\left(\xi^{1}, \xi^{2}\right)$. Moreover, the Weyl projection is compatible with the reality condition (A.11) and we may therefore build symplectic Majorana-Weyl solutions to (B.4) using the chirality projectors

$$
\xi_{ \pm}^{i}=\frac{\left(1 \pm \gamma_{5}\right)}{2} \xi^{i}
$$

The procedure just described builds eight linearly independent, symplectic Majorana-Weyl solutions to (B.1), (B.2), and these solutions all have $\eta_{ \pm}^{i}=0$.

As explained in the introduction, in the context of localization we are interested in the supercharge which squares to $\left(L_{0}-J_{0}\right)$. This supercharge is parametrized by $\xi_{(1) \pm}^{i}$ 
of (B.10), whose Weyl-projected $\mathrm{SU}(2)$ components are explicitly given by

$$
\begin{aligned}
& \xi_{(1)+}^{1}=\frac{e^{-\frac{i}{2}(\tau+\phi)}}{\sqrt{2}}\left(\begin{array}{c}
i \cosh \frac{\eta}{2} \sin \frac{\psi}{2} \\
0 \\
0 \\
\sinh \frac{\eta}{2} \cos \frac{\psi}{2}
\end{array}\right), \quad \xi_{(1)+}^{2}=\frac{e^{\frac{i}{2}(\tau+\phi)}}{\sqrt{2}}\left(\begin{array}{c}
i \sinh \frac{\eta}{2} \cos \frac{\psi}{2} \\
0 \\
0 \\
0 \\
-\cosh \frac{\eta}{2} \sin \frac{\psi}{2}
\end{array}\right), \\
& 0 \\
& \xi_{(1)-}^{1}=\frac{e^{-\frac{i}{2}(\tau+\phi)}}{\sqrt{2}}\left(\begin{array}{c}
-\sinh \frac{\eta}{2} \sin \frac{\psi}{2} \\
i \cosh \frac{\eta}{2} \cos \frac{\psi}{2} \\
0
\end{array}\right), \quad \xi_{(1)-}^{2}=\frac{e^{\frac{i}{2}(\tau+\phi)}}{\sqrt{2}}\left(\begin{array}{c}
-\cosh \frac{\eta}{2} \cos \frac{\psi}{2} \\
-i \sinh \frac{\eta}{2} \sin \frac{\psi}{2} \\
0
\end{array}\right) .
\end{aligned}
$$

The supercharge $Q$ built out of this spinor satisfies the algebra (2.27). The fermionic bilinears

$$
v^{\mu}=-2 i \epsilon_{i j} \bar{\xi}_{+}^{i} \gamma^{\mu} \xi_{-}^{j}, \quad K_{ \pm}=\epsilon_{i j} \bar{\xi}_{ \pm}^{i} \xi_{ \pm}^{j}
$$

that are used in the main text, have the following expressions:

$$
v^{\mu}=\left(\begin{array}{llll}
-1 & 0 & 0 & 1
\end{array}\right)^{T}
$$

and

$$
K_{ \pm}=\frac{1}{2}( \pm \cos \psi-\cosh \eta)
$$

\section{Transversally elliptic operators and the symbol of $D_{10}$}

The standard starting point for the considerations of index theory is that of an elliptic operator on a manifold, which generalizes the notion of a Laplacian. If the operator is linear and of second order, we can write it in local coordinates $x^{i}$ as

$$
a^{i j}(x) \partial_{i} \partial_{j}+b^{i}(x) \partial_{i}+c_{i}(x)
$$

An elliptic operator is one for which the matrix $a^{i j}$ is positive-definite. ${ }^{25}$ This can be restated as follows: if we replace the derivatives by momenta, i.e. consider the Fourier transform of the linear operator, we obtain the symbol of the operator. An operator is elliptic if the principal symbol $a^{i j} p_{i} p_{j}$ does not vanish for any non-zero $p_{i}$.

Our operator $D_{10}$, however, is not elliptic - but it can still be treated by index theory [50]. The point is that we have a certain special $\mathrm{U}(1)$ action (that of $H$ ), and our operator $D_{10}$ commutes with this action. In the directions transverse to the $\mathrm{U}(1)$ orbits, the operator $D_{10}$ is elliptic - such an operator is called transversally elliptic, and there is a version of the index theorem that deals with such operators. In terms of the symbol, an operator is called transversally elliptic if its symbol does not vanish for any $p_{i}$ that is transversal to the generator of the $\mathrm{U}(1)$ action. This means that the matrix $a_{i j}$ is allowed to degenerate, but only along the one-dimensional locus generated by the U(1) action.

\footnotetext{
${ }^{25}$ For technical reasons, the theory of elliptic operators often also assumes that the eigenvalues are bounded.
} 
We show below that the operator $D_{10}$ is transversally elliptic with respect to the $\mathrm{U}(1)$ symmetry generated by $H$.

As explained in section 4, the one-loop determinant acquires contributions from vector and hyper multiplets separately. We therefore need to prove that the operators $D_{10}^{\text {vect }}$ in the vector multiplet sector and $D_{10}^{\text {hyp }}$ in the hyper multiplet sector are both transversally elliptic with respect to the $\mathrm{U}(1)$ action generated by $H$.

\section{Vector multiplets}

To read off the form of the operator $D_{10}^{\text {vect }}$, we introduce the following quantities:

$$
\begin{aligned}
\Lambda^{I} & \equiv Q\left(X^{I}-\bar{X}^{I}\right)=\epsilon_{i j}\left(\bar{\xi}_{+}^{i} \lambda_{+}^{I j}-\bar{\xi}_{-}^{i} \lambda_{-}^{I j}\right), \\
\Lambda_{\mu}^{I} & \equiv Q A_{\mu}^{I}=\epsilon_{i j}\left(\bar{\xi}_{-}^{i} \gamma_{\mu} \lambda_{+}^{I j}-\bar{\xi}_{+}^{i} \gamma_{\mu} \lambda_{-}^{I j}\right), \\
\Xi^{I i j} & \left.\equiv 2\left(\bar{\xi}_{+}^{(i} \lambda_{+}^{I j}\right)+\bar{\xi}_{-}^{(i} \lambda_{-}^{I j j}\right) .
\end{aligned}
$$

We split the fields of the vector multiplet (including the BRST ghosts) into bosons and fermions according to

$$
X=\left\{A_{\mu}^{I}, X^{I}-\bar{X}^{I}\right\}, \quad \Psi=\left\{\Xi^{I i j}, b^{I}, c^{I}\right\},
$$

and their $\widehat{Q}$-superpartners

$$
\widehat{Q} \Psi=\left\{\widehat{Q} \Xi^{I i j}, B^{I},-\widehat{\theta}^{I}\right\}, \quad \widehat{Q} X=\left\{\widehat{Q} A_{\mu}, \widehat{Q}\left(X^{I}-\bar{X}^{I}\right)\right\} .
$$

The relations (C.2) may be inverted to yield

$$
\begin{aligned}
& \lambda_{+}^{I i}=\frac{-1}{\cosh \eta}\left(\xi_{+}^{i} \Lambda^{I}+\gamma^{\mu} \xi_{-}^{i} \Lambda_{\mu}^{I}-\epsilon_{k l} \xi_{+}^{k} \Xi^{I i l}\right), \\
& \lambda_{-}^{I i}=\frac{1}{\cosh \eta}\left(\xi_{-}^{i} \Lambda^{I}+\gamma^{\mu} \xi_{+}^{i} \Lambda_{\mu}^{I}+\epsilon_{k l} \xi_{-}^{k} \Xi^{I i l}\right) .
\end{aligned}
$$

The deformation operator in the vector multiplet sector, $\widehat{\mathcal{V}}^{\text {vect }}$, is as follows:

$$
\widehat{\mathcal{V}}^{\mathrm{vect}}=\int d^{4} x \sum_{I}\left[\left(\widehat{Q} \lambda_{+}^{i}, \lambda_{+}^{I i}\right)+\left(\widehat{Q} \lambda_{-}^{i}, \lambda_{-}^{I i}\right)+b^{I} G^{A}\left(A_{\mu}^{I}\right)\right],
$$

As explained in appendix A, we will take the inner product in the equation above to be the hermitian conjugate in Minkowski signature, compute the quantity in the right-hand side using this inner product and the original Minkowski reality conditions on the fields, and only conduct the Euclidean continuation at the very end by imposing different reality conditions on the various fields. Thus, we write:

$$
\widehat{\mathcal{V}}^{\mathrm{vect}}=\int d^{4} x \sum_{I}\left[\left(\widehat{Q} \lambda_{+}^{i}\right)^{\dagger} \lambda_{+}^{I i}+\left(\widehat{Q} \lambda_{-}^{i}\right)^{\dagger} \lambda_{-}^{I i}+b^{I} G^{A}\left(A_{\mu}^{I}\right)\right],
$$

In terms of the variables (C.2), this is

$\widehat{\mathcal{V}}^{\text {vect }}=\int d^{4} x \sum_{I}\left[\frac{1}{\cosh \eta}\left(\left(\widehat{Q} \Lambda^{I}\right)^{\dagger} \Lambda^{I}+\left(\widehat{Q} \Lambda_{\mu}^{I}\right)^{\dagger} \Lambda^{I \mu}+\frac{1}{2}\left(\widehat{Q} \Xi^{I i j}\right)^{\dagger} \Xi^{I i j}\right)+b^{I} G^{A}\left(A_{\mu}^{I}\right)\right]$. 
To compare to the general expression for the quadratic fluctuations (4.3), we express the regulator in terms of the fields (C.3), (C.4). We write the various terms in the equation above in terms of these fields by making use of the following relations:

$$
\begin{array}{ll}
\Lambda^{I}=\widehat{Q}\left(X^{I}-\bar{X}^{I}\right), & \left(\widehat{Q} \Lambda^{I}\right)^{\dagger}=i v^{\mu} \partial_{\mu}\left(X^{I}-\bar{X}^{I}\right), \\
\Lambda_{\mu}^{I}=\widehat{Q} A_{\mu}^{I}-\partial_{\mu} c^{I}, & \left(\widehat{Q} \Lambda_{\mu}^{I}\right)^{\dagger}=-i v^{\nu} F_{\nu \mu}^{I}+i v^{\nu} \partial_{\mu} A_{\nu}^{I}+2 \partial_{\mu}\left(\cos \psi\left(X^{I}-\bar{X}^{I}\right)\right)-\partial_{\mu} \widehat{Q} c^{I} .
\end{array}
$$

Here we use the Minkowksi reality conditions on the vector multiplet fields:

$$
\left(X^{I}\right)^{\dagger}=\bar{X}^{I} \quad\left(A_{\mu}^{I}\right)^{\dagger}=A_{\mu}^{I},
$$

as consistent with the original $\mathcal{N}=2$ Minkowski superconformal algebra.

Further, we have:

$$
\widehat{Q} \Xi^{I i j}=\left(\bar{\xi}_{+}^{(i} \gamma^{a b} \xi_{+}^{j)}+\bar{\xi}_{-}^{(i} \gamma^{a b} \xi_{-}^{j)}\right) \mathcal{F}_{a b}^{I}+4 \bar{\xi}_{+}^{(i} \gamma^{\mu} \xi_{-}^{j)} \partial_{\mu}\left(X^{I}-\bar{X}^{I}\right)+2\left(\bar{\xi}_{+}^{k} \xi_{+}^{(i}+\bar{\xi}_{-}^{k} \xi_{-}^{(i}\right) Y_{k}^{I j)}
$$

where $\mathcal{F}_{a b}^{I}=F_{a b}^{I}-\frac{1}{4}\left(\bar{X}^{I} T_{a b}^{-}+X^{I} T_{a b}^{+}\right)$, and we can use this equation to express the auxiliary $Y_{i j}^{I}$ in terms of the cohomological fields as follows:

$$
2\left(\bar{\xi}_{+}^{k} \xi_{+}^{(j}+\bar{\xi}_{-}^{k} \xi_{-}^{(j}\right) Y_{k}^{I l)}=\widehat{Q} \Xi^{I j l}-\left(\bar{\xi}_{+}^{(j} \gamma^{a b} \xi_{+}^{l)}+\bar{\xi}_{-}^{(j} \gamma^{a b} \xi_{-}^{l)}\right) \mathcal{F}_{a b}^{I}-4 \bar{\xi}_{+}^{(j} \gamma^{\mu} \xi_{-}^{l)} \partial_{\mu}\left(X^{I}-\bar{X}^{I}\right) .
$$

From this we deduce

$$
\begin{aligned}
\left(\widehat{Q} \Xi^{I i j}\right)^{\dagger}= & \epsilon_{i k} \epsilon_{j l}\left[\widehat{Q} \Xi^{I k l}-2\left(\bar{\xi}_{+}^{(k} \gamma^{a b} \xi_{+}^{l)}+\bar{\xi}_{-}^{(k} \gamma^{a b} \xi_{-}^{l)}\right) F_{a b}^{I}-8 \bar{\xi}_{+}^{(k} \gamma^{\mu} \xi_{-}^{l)} \partial_{\mu}\left(X^{I}-\bar{X}^{I}\right)\right] \\
& +\frac{1}{4} \epsilon_{i k} \epsilon_{j l}\left(\bar{\xi}_{+}^{(k} \gamma^{a b} \xi_{+}^{l)}+\bar{\xi}_{-}^{(k} \gamma^{a b} \xi_{-}^{l)}\right)\left(T_{a b}^{+}-T_{a b}^{-}\right)\left(X^{I}-\bar{X}^{I}\right) .
\end{aligned}
$$

Once these expressions have been derived, we can analytically continue to Euclidean by imposing the real slice in which $X$ and $\bar{X}$ are independent real variables.

Collecting all this, we can write the terms of the regulator $\widehat{\mathcal{V}}^{\text {vect }}$ relevant for the symbol computation in each vector multiplet $I$ :

$$
\begin{aligned}
& i \partial^{\mu} c^{I} v^{\nu} F_{\nu \mu}^{I}-i \partial^{\mu} c^{I} v^{\nu} \partial_{\mu} A_{\nu}^{I}-2 \partial^{\mu} c^{I} \partial_{\mu}\left(\cos \psi\left(X^{I}-\bar{X}^{I}\right)\right)+b^{I} G^{A}\left(A_{\mu}^{I}\right) \\
& -\epsilon_{i k} \epsilon_{j l} \Xi^{I i j}\left(\bar{\xi}_{+}^{(k} \gamma^{a b} \xi_{+}^{l)}+\bar{\xi}_{-}^{(k} \gamma^{a b} \xi_{-}^{l)}\right) F_{a b}^{I}-4 \epsilon_{i k} \epsilon_{j l} \Xi^{I i j} \bar{\xi}_{-}^{(k} \gamma^{\mu} \xi_{+}^{l)} \partial_{\mu}\left(X^{I}-\bar{X}^{I}\right) .
\end{aligned}
$$

We are interested in the symbol of the $D_{10}^{\text {vect }}$ operator, $\sigma\left(D_{10}^{\text {vect }}\right)$, which is obtained by replacing derivatives according to $\partial_{a} \rightarrow i p_{a}$, where $p_{a}$ are momenta. In order for the index theorem to apply, we wish to verify that $D_{10}^{\text {vect }}$ is transversally elliptic with respect to the $\mathrm{U}(1)$ symmetry generated by $H$. Therefore, we want to check that the determinant of its symbol is non-zero everywhere in $A d S_{2} \times S^{2}$ as long as momenta transverse to the direction specified by $v^{\mu}$ are turned on. We still have some freedom in choosing the gaugefixing function $G^{A}$. If we take the usual Lorentz gauge

$$
G\left(A_{\mu}^{I}\right)=\partial^{\mu} A_{\mu}^{I},
$$


we find that the determinant of the symbol in each vector multiplet $I$ is given by

$$
\operatorname{det}\left[\sigma\left(D_{10}^{\text {vect }}\right)\right]=-2\left(p_{i}^{2}+p_{v}^{2}\right)\left(p_{i}^{2} \cosh ^{2} \eta+p_{v}^{2} \cos ^{2} \psi\right)^{2},
$$

where $p_{i}, i=1,2,3$, are momenta transverse to the $H$ action and $p_{v}$ is the momenta along $v^{\mu}$. We clearly see that, when $p_{i}=0$, the determinant vanishes on the equator of the $S^{2}$. When some transverse momentum is turned on, this degeneracy is lifted and the determinant of the symbol is non-zero everywhere in space-time. This shows that the operator $D_{10}^{\text {vect }}$ for each vector multiplet $I$ is indeed transversally elliptic.

\section{Hyper multiplets}

In the hyper multiplet sector, we introduce

$$
\begin{aligned}
& \Lambda_{i}^{\alpha} \equiv Q A_{i}^{\alpha}=2 \epsilon_{i j}\left(\bar{\xi}_{-}^{j} \zeta_{-}^{\alpha}-\bar{\xi}_{+}^{j} \zeta_{+}^{\alpha}\right) \\
& \Xi_{i}^{\alpha} \equiv 2 \epsilon_{i j}\left(\overline{\breve{\xi}}_{+}^{j} \zeta_{+}^{\alpha}+\overline{\bar{\xi}}_{-}^{j} \zeta_{-}^{\alpha}\right)
\end{aligned}
$$

We split the fields according to

$$
X=\left\{A_{i}^{\alpha}\right\}, \quad \Psi=\left\{\Xi_{i}^{\alpha}\right\},
$$

and their $\widehat{Q}$-superpartners. We can once again invert the relations (C.18) to write

$$
\zeta_{+}^{\alpha}=\frac{-1}{\cosh \eta}\left(\Lambda_{i}^{\alpha} \xi_{+}^{i}-\Xi_{i}^{\alpha} \breve{\xi}_{+}^{i}\right), \quad \zeta_{-}^{\alpha}=\frac{1}{\cosh \eta}\left(\Lambda_{i}^{\alpha} \xi_{-}^{i}+\Xi_{i}^{\alpha} \breve{\xi}_{-}^{i}\right)
$$

The deformation operator for one hyper multiplet is now

$$
\widehat{\mathcal{V}}^{\text {hyp }}=\int d^{4} x\left[\left(\widehat{Q} \zeta_{+}^{\alpha}\right)^{\dagger} \zeta_{+}^{\alpha}+\left(\widehat{Q} \zeta_{-}^{\alpha}\right)^{\dagger} \zeta_{-}^{\alpha}\right]
$$

In terms of the variables (C.18), this is

$$
\widehat{\mathcal{V}}^{\text {hyp }}=\int d^{4} x \frac{1}{2 \cosh \eta}\left[\left(\widehat{Q} \Lambda_{i}^{\alpha}\right)^{\dagger} \Lambda_{i}^{\alpha}+\left(\widehat{Q} \Xi_{i}^{\alpha}\right)^{\dagger} \Xi_{i}^{\alpha}\right]
$$

We have

$$
\left(\widehat{Q} \Xi_{i}^{\alpha}\right)^{\dagger}=\widehat{Q} \Xi_{\alpha}^{i}+4 \rho_{\alpha \beta}\left(\overline{\breve{\xi}}_{+}^{i} \gamma^{\mu} \xi_{-}^{j}+\overline{\breve{\xi}}_{-}^{i} \gamma^{\mu} \xi_{+}^{j}\right) \partial_{\mu} A_{j}^{\beta},
$$

where we've again made use of the Minkowski reality condition

$$
\left(A_{i}^{\alpha}\right)^{\dagger}=\epsilon^{i j} \rho_{\alpha \beta} A_{j}^{\beta} .
$$

We see that the relevant term for the symbol of $D_{10}^{\text {hyp }}$ is simply

$$
4 \rho_{\alpha \beta} \Xi_{i}^{\alpha}\left(\overline{\breve{\xi}}_{+}^{i} \gamma^{\mu} \xi_{-}^{j}+\bar{\xi}_{-}^{i} \gamma^{\mu} \xi_{+}^{j}\right) \partial_{\mu} A_{j}^{\beta}
$$


To compute the symbol and its determinant, we therefore need an explicit solution to the constraint equations (2.25). We choose:

$$
\breve{\xi}_{+}^{i}=\left(\frac{\cosh \eta-\cos \psi}{\cosh \eta+\cos \psi}\right)^{-1 / 2} \xi_{+}^{i}, \quad \breve{\xi}_{-}^{i}=\left(\frac{\cosh \eta-\cos \psi}{\cosh \eta+\cos \psi}\right)^{1 / 2} \xi_{-}^{i},
$$

and we find for the determinant of the symbol of $D_{10}^{\text {hyp }}$

$$
\operatorname{det}\left[\sigma\left(D_{10}^{\mathrm{hyp}}\right)\right]=2\left(p_{i}^{2} \cosh ^{2} \eta+p_{v}^{2} \cos ^{2} \psi\right)
$$

where we use the momenta and notation introduced for the vector multiplet case. We again see that the symbol is non-invertible along the equator of $S^{2}$ when only the momentum along the $H$ action is non-zero, and this degeneracy is lifted whenever some transverse momenta are turned on. Thus the operator $D_{10}^{\text {hyp }}$ is transversally elliptic with respect to the $H$ action.

Open Access. This article is distributed under the terms of the Creative Commons Attribution License (CC-BY 4.0), which permits any use, distribution and reproduction in any medium, provided the original author(s) and source are credited.

\section{References}

[1] S. Ferrara, R. Kallosh and A. Strominger, $\mathcal{N}=2$ extremal black holes, Phys. Rev. D 52 (1995) 5412 [hep-th/9508072] [INSPIRE].

[2] J.D. Bekenstein, Black holes and entropy, Phys. Rev. D 7 (1973) 2333 [InSPIRE].

[3] S.W. Hawking, Particle Creation by Black Holes, Commun. Math. Phys. 43 (1975) 199 [Erratum ibid. 46 (1976) 206] [INSPIRE].

[4] G. Lopes Cardoso, B. de Wit and T. Mohaupt, Corrections to macroscopic supersymmetric black hole entropy, Phys. Lett. B 451 (1999) 309 [hep-th/9812082] [INSPIRE].

[5] G. Lopes Cardoso, B. de Wit, J. Kappeli and T. Mohaupt, Stationary BPS solutions in $N=2$ supergravity with $R^{2}$ interactions, JHEP 12 (2000) 019 [hep-th/0009234] [INSPIRE].

[6] R.M. Wald, Black hole entropy is the Noether charge, Phys. Rev. D 48 (1993) 3427 [gr-qc/9307038] [INSPIRE].

[7] V. Iyer and R.M. Wald, Some properties of Noether charge and a proposal for dynamical black hole entropy, Phys. Rev. D 50 (1994) 846 [gr-qc/9403028] [INSPIRE].

[8] T. Jacobson, G. Kang and R.C. Myers, Black hole entropy in higher curvature gravity, gr-qc/9502009 [INSPIRE].

[9] A. Sen, Black hole entropy function and the attractor mechanism in higher derivative gravity, JHEP 09 (2005) 038 [hep-th/0506177] [INSPIRE].

[10] A. Sen, Quantum Entropy Function from $A d S_{2} / C F T_{1}$ Correspondence, Int. J. Mod. Phys. A 24 (2009) 4225 [arXiv:0809.3304] [INSPIRE].

[11] N. Banerjee, S. Banerjee, R.K. Gupta, I. Mandal and A. Sen, Supersymmetry, Localization and Quantum Entropy Function, JHEP 02 (2010) 091 [arXiv:0905.2686] [INSPIRE]. 
[12] A. Dabholkar, J. Gomes and S. Murthy, Quantum black holes, localization and the topological string, JHEP 06 (2011) 019 [arXiv: 1012.0265] [INSPIRE].

[13] A. Dabholkar, J. Gomes and S. Murthy, Localization \& Exact Holography, JHEP 04 (2013) 062 [arXiv: 1111.1161] [INSPIRE].

[14] R.K. Gupta and S. Murthy, All solutions of the localization equations for $N=2$ quantum black hole entropy, JHEP 02 (2013) 141 [arXiv:1208.6221] [INSPIRE].

[15] S. Murthy and V. Reys, Quantum black hole entropy and the holomorphic prepotential of $N=2$ supergravity, JHEP 10 (2013) 099 [arXiv:1306.3796] [INSPIRE].

[16] A. Dabholkar, J. Gomes and S. Murthy, Nonperturbative black hole entropy and Kloosterman sums, JHEP 03 (2015) 074 [arXiv: 1404.0033] [INSPIRE].

[17] V. Pestun, Localization of gauge theory on a four-sphere and supersymmetric Wilson loops, Commun. Math. Phys. 313 (2012) 71 [arXiv:0712.2824] [INSPIRE].

[18] V. Pestun, Localization for $\mathcal{N}=2$ Supersymmetric Gauge Theories in Four Dimensions, Math. Phys. Stud. 2016 (2016) 159 [arXiv:1412.7134] [INSPIRE].

[19] K. Hosomichi, The localization principle in SUSY gauge theories, PTEP 2015 (2015) 11B101 [arXiv: 1502.04543] [INSPIRE].

[20] M.F. Atiyah and R. Bott, The moment map and equivariant cohomology, Topology 23 (1984) 1 [INSPIRE].

[21] J.J. Duistermaat and G.J. Heckman, On the Variation in the cohomology of the symplectic form of the reduced phase space, Invent. Math. 69 (1982) 259 [INSPIRE].

[22] N. Berline and M. Vergne, Classes caractéristiques équivariantes. Formule de localisation en cohomologie équivariante, C.R. Acad. Sci. Paris Sér. I Math. 295 (1982) 539.

[23] B. de Wit, J.W. van Holten and A. Van Proeyen, Transformation Rules of $N=2$ Supergravity Multiplets, Nucl. Phys. B 167 (1980) 186 [InSPIRE].

[24] B. de Wit, J.W. van Holten and A. Van Proeyen, Structure of $N=2$ Supergravity, Nucl. Phys. B 184 (1981) 77 [Erratum ibid. B 222 (1983) 516] [inSPIRE].

[25] N. Banerjee, D.P. Jatkar and A. Sen, Asymptotic Expansion of the $N=4$ Dyon Degeneracy, JHEP 05 (2009) 121 [arXiv:0810.3472] [INSPIRE].

[26] S. Murthy and B. Pioline, A Farey tale for $N=4$ dyons, JHEP 09 (2009) 022 [arXiv: 0904.4253] [INSPIRE].

[27] H. Ooguri, A. Strominger and C. Vafa, Black hole attractors and the topological string, Phys. Rev. D 70 (2004) 106007 [hep-th/0405146] [INSPIRE].

[28] F. Denef and G.W. Moore, Split states, entropy enigmas, holes and halos, JHEP 11 (2011) 129 [hep-th/0702146] [INSPIRE].

[29] G.L. Cardoso, B. de Wit and S. Mahapatra, Subleading and non-holomorphic corrections to $N=2$ BPS black hole entropy, JHEP 02 (2009) 006 [arXiv:0808.2627] [INSPIRE].

[30] B. de Wit, S. Murthy and V. Reys, in preparation.

[31] I.A. Batalin and G.A. Vilkovisky, Gauge Algebra and Quantization, Phys. Lett. B 102 (1981) 27 [INSPIRE].

[32] B. de Wit and J.W. van Holten, Covariant Quantization of Gauge Theories with Open Gauge Algebra, Phys. Lett. B 79 (1978) 389 [INSPIRE]. 
[33] N. Hama and K. Hosomichi, Seiberg-Witten Theories on Ellipsoids, JHEP 09 (2012) 033 [arXiv: 1206.6359] [INSPIRE].

[34] A. Dabholkar, F. Denef, G.W. Moore and B. Pioline, Precision counting of small black holes, JHEP 10 (2005) 096 [hep-th/0507014] [INSPIRE].

[35] B. Pioline, Lectures on black holes, topological strings and quantum attractors, Class. Quant. Grav. 23 (2006) S981 [hep-th/0607227] [INSPIRE].

[36] R.K. Gupta, Y. Ito and I. Jeon, Supersymmetric Localization for BPS Black Hole Entropy: 1-loop Partition Function from Vector Multiplets, arXiv:1504.01700 [INSPIRE].

[37] J. Gomes, Quantum entropy and exact 4d/5d connection, JHEP 01 (2015) 109 [arXiv: 1305.2849] [INSPIRE].

[38] B. Sahoo and A. Sen, Higher derivative corrections to non-supersymmetric extremal black holes in $N=2$ supergravity, JHEP 09 (2006) 029 [hep-th/0603149] [INSPIRE].

[39] S. Ferrara and R. Kallosh, Supersymmetry and attractors, Phys. Rev. D 54 (1996) 1514 [hep-th/9602136] [INSPIRE].

[40] A. Van Proeyen, Tools for supersymmetry, Ann. U. Craiova Phys. 9 (1999) I.1-I.48 [hep-th/9910030] [INSPIRE].

[41] C. Klare and A. Zaffaroni, Extended Supersymmetry on Curved Spaces, JHEP 10 (2013) 218 [arXiv: 1308.1102] [INSPIRE].

[42] B. de Wit, P.G. Lauwers and A. Van Proeyen, Lagrangians of $N=2$ Supergravity - Matter Systems, Nucl. Phys. B 255 (1985) 569 [INSPIRE].

[43] N. Berkovits, A ten-dimensional super Yang-Mills action with off-shell supersymmetry, Phys. Lett. B 318 (1993) 104 [hep-th/9308128] [INSPIRE].

[44] L.F. Alday, D. Martelli, P. Richmond and J. Sparks, Localization on Three-Manifolds, JHEP 10 (2013) 095 [arXiv: 1307.6848] [INSPIRE].

[45] J. Gomis, J. Paris and S. Samuel, Antibracket, antifields and gauge theory quantization, Phys. Rept. 259 (1995) 1 [hep-th/9412228] [INSPIRE].

[46] A. Castro, D. Grumiller, F. Larsen and R. McNees, Holographic Description of AdS $S_{2}$ Black Holes, JHEP 11 (2008) 052 [arXiv:0809.4264] [INSPIRE].

[47] A. Sen, Logarithmic Corrections to $N=2$ Black Hole Entropy: An Infrared Window into the Microstates, Gen. Rel. Grav. 44 (2012) 1207 [arXiv:1108.3842] [INSPIRE].

[48] M. Henningson and K. Sfetsos, Spinors and the AdS/CFT correspondence, Phys. Lett. B 431 (1998) 63 [hep-th/9803251] [INSPIRE].

[49] S. Lee, Index, supersymmetry and localization, lectures at the Pyeong-Chang Summer School, http://psi.kias.re.kr/2013/sub02/sub02_01.php, 2013.

[50] M.F. Atiyah, Elliptic operators and compact groups, Lecture Notes in Mathematics, Springer Verlag, Volume 401, (1974).

[51] S.W. Hawking, Zeta Function Regularization of Path Integrals in Curved Space-Time, Commun. Math. Phys. 55 (1977) 133 [InSPIRE].

[52] S. Banerjee, R.K. Gupta and A. Sen, Logarithmic Corrections to Extremal Black Hole Entropy from Quantum Entropy Function, JHEP 03 (2011) 147 [arXiv: 1005.3044] [INSPIRE]. 
[53] S. Banerjee, R.K. Gupta, I. Mandal and A. Sen, Logarithmic Corrections to $N=4$ and $N=8$ Black Hole Entropy: A One Loop Test of Quantum Gravity, JHEP 11 (2011) 143 [arXiv:1106.0080] [INSPIRE].

[54] C. Keeler, F. Larsen and P. Lisbao, Logarithmic Corrections to $N \geq 2$ Black Hole Entropy, Phys. Rev. D 90 (2014) 043011 [arXiv: 1404.1379] [InSPIRE].

[55] F. Larsen and P. Lisbao, Quantum Corrections to Supergravity on $A d S_{2} \times S^{2}$, Phys. Rev. D 91 (2015) 084056 [arXiv: 1411.7423] [InSPIRE].

[56] D.V. Vassilevich, Heat kernel expansion: User's manual, Phys. Rept. 388 (2003) 279 [hep-th/0306138] [INSPIRE].

[57] D. Shih and X. Yin, Exact black hole degeneracies and the topological string, JHEP 04 (2006) 034 [hep-th/0508174] [inSPIRE].

[58] A. Dabholkar, J. Gomes, S. Murthy and V. Reys, in preparation.

[59] G. Lopes Cardoso, B. de Wit, J. Kappeli and T. Mohaupt, Black hole partition functions and duality, JHEP 03 (2006) 074 [hep-th/0601108] [INSPIRE].

[60] E.P. Verlinde, Attractors and the holomorphic anomaly, hep-th/0412139 [INSPIRE].

[61] G.L. Cardoso, B. de Wit and S. Mahapatra, Deformations of special geometry: in search of the topological string, JHEP 09 (2014) 096 [arXiv: 1406.5478] [INSPIRE].

[62] M. Dedushenko and E. Witten, Some Details On The Gopakumar-Vafa and Ooguri-Vafa Formulas, arXiv: 1411.7108 [INSPIRE].

[63] E. Kiritsis and B. Pioline, On $R^{4}$ threshold corrections in IIB string theory and $(p, q)$ string instantons, Nucl. Phys. B 508 (1997) 509 [hep-th/9707018] [INSPIRE].

[64] M.B. Green, J.G. Russo and P. Vanhove, String theory dualities and supergravity divergences, JHEP 06 (2010) 075 [arXiv: 1002.3805] [INSPIRE].

[65] A. Sen, Arithmetic of Quantum Entropy Function, JHEP 08 (2009) 068 [arXiv:0903.1477] [INSPIRE].

[66] A. Dabholkar, J. Gomes, S. Murthy and A. Sen, Supersymmetric Index from Black Hole Entropy, JHEP 04 (2011) 034 [arXiv:1009.3226] [INSPIRE].

[67] A. Dabholkar, S. Murthy and D. Zagier, Quantum Black Holes, Wall Crossing and Mock Modular Forms, arXiv:1208.4074 [INSPIRE].

[68] J.A. Harvey and G.W. Moore, Algebras, BPS states and strings, Nucl. Phys. B 463 (1996) 315 [hep-th/9510182] [INSPIRE].

[69] J.A. Harvey and G.W. Moore, On the algebras of BPS states, Commun. Math. Phys. 197 (1998) 489 [hep-th/9609017] [INSPIRE].

[70] H. Lü, C.N. Pope and J. Rahmfeld, A construction of Killing spinors on $S^{n}$, J. Math. Phys. 40 (1999) 4518 [hep-th/9805151] [INSPIRE]. 